\title{
On the convergence of stationary sequences in topology optimization*
}

\author{
Anton Evgrafov ${ }^{\dagger}$ and Michael Patriksson ${ }^{\ddagger}$
}

January 14, 2005

\begin{abstract}
We consider structural topology optimization problems including unilateral constraints arising from non-penetration conditions in contact mechanics. The resulting non-convex non-smooth problems are instances of mathematical programs with equilibrium constraints (MPEC), or bi-level programs. Applying nested (implicit programming) algorithms to this class of problems is problematic owing to the singularity of the feasible set. We propose a perturbation strategy combining the relaxation of the equilibrium constraint with the restriction of the design domain to its regular part only. This strategy allows us to attack the problem numerically using standard nonlinear programming algorithms.

We rigorously study the optimality conditions for the original singular problem as well as the convergence of stationary points and globally optimal solutions to approximating problems towards respectively stationary points and globally optimal solutions to the original problem. A limited numerical benchmarking of the algorithm is performed.
\end{abstract}

Keywords: topology optimization, $\varepsilon$-perturbation, local optimality, stress singularity, MPEC, smoothing

\section{Introduction}

The optimum design of trusses is concerned with the distribution of the available material among structural members (bars) in order to carry a given set of loads as efficiently as possible, subject to mechanical and technological constraints. The distinguishing feature of structural optimization problems is the presence of the complicating equilibrium constraint, relating design variables (i.e., those controlling the material distribution) with state variables (e.g., nodal displacements and stresses in the structural members). Verbally, the relation between the two sets of variables can be formulated as follows: the state variables solve a parametric optimization problem with design variables as parameters. Therefore, the problem belongs to a class of difficult optimization problems known as mathematical programs with equilibrium constraints (MPEC), or generalized bi-level programming problems.

In the framework of topology optimization (as opposed to sizing optimization), the topology of a truss may change as a result of the optimization process, that is, if a zero amount of material is allocated to some parts; this possibility significantly enlarges the design space and, at the same time, increases the computational complexity of the problem. The former implies the possibility to obtain optimal designs that perform much better than their "sizing" counterparts [Mic04]; the latter places significant requirements on algorithms for solving topology optimization problems.

In most cases, "standard" algorithms for differentiable nonlinear programming problems can be applied directly to sizing optimization problems. Therefore, one natural approach to topology optimization is to introduce a small but positive lower bound $\varepsilon$ on the bar volumes, thus converting the problem into a sizing one. Solving a sequence of sizing problems for $\varepsilon$ converging to zero produces a sequence of designs, whose limit points one hopes are optimal in the original topology optimization problem.

Unfortunately, some important constraints produce design domains that violate standard nonlinear programming constraint qualifications; in particular, some optimal solutions cannot be reached as limits of any sequence of strictly positive feasible designs. The stress singularity phenomenon appearing in topology optimization problems with constraints on the maximal effective stresses in the structural members is probably the one most studied and the one that has attracted the most recent interest-we mention the

\footnotetext{
*This research is supported by the Swedish Research Council (grant 621-2002-5780).

${ }^{\dagger}$ Department of Mathematics, Chalmers University of Technology, SE-412 96 Göteborg, Sweden, toxa@math.chalmers.se

${ }^{\ddagger}$ Department of Mathematics, Chalmers University of Technology, SE-412 96 Göteborg, Sweden, mipat@math.chalmers . se
} 
work in [SvG68, ChG97, Pet01, StS01], just to name a few references. Similarly, local (Euler) buckling constraints [GCY01], and global (system) stability constraints [Evg04] are known to exhibit a singular behaviour.

Sizing approximations, studied in the cited papers, are all concerned with approximations of the globally optimal solutions. In computational practice, however, it is very difficult to solve the non-convex approximating problems to global optimality. Since most numerical nonlinear optimization algorithms can only find stationary points of the approximating sizing problems, in this paper we study the limit points of such sequences. We show that they are indeed stationary, in some sense, in the limiting (that is, original) topology optimization problem as well.

\subsection{Equilibrium problem}

We consider a truss with $m$ bars and $n$ degrees of freedom. There are $r$ designated nodes of the truss that may come into frictionless unilateral contact with rigid obstacles.

Given positions of the nodes, the design (and topology in particular) of a truss can be described by prescribing for each bar $i, i=1, \ldots, m$, the amount of material $x_{i} \geq 0$ allocated to this bar. For convenience we collect all the design variables in a vector $\boldsymbol{x}=\left(x_{1}, \ldots, x_{m}\right)^{t} \in \mathbb{R}_{+}^{m}$. We introduce an index set of the present (or, active) members in the structure $\mathcal{I}(\boldsymbol{x})=\left\{i=1, \ldots, m \mid x_{i}>0\right\}$, and denote by $\mathcal{I}^{c}(\boldsymbol{x})$ the complement of $\mathcal{I}(\boldsymbol{x})$ in $\{1, \ldots, m\}$.

Given a particular design $\boldsymbol{x}$, the equilibrium status of a truss can be described by specifying

- a pseudo-force $s_{i}$ (also known as the normalized stress, which is in fact a stress in the bar times its volume) for each bar $i \in \mathcal{I}(\boldsymbol{x})$ present in the structure. To simplify the notation we collect all values $s_{i}$, $i=1, \ldots, m$, into one vector $s \in \mathbb{R}^{m}$, assuming $s_{i}=0$ for $i \notin \mathcal{I}(\boldsymbol{x})$;

- a contact force $\lambda_{j}$ for each of the potential contact nodes $j=1, \ldots, r$. These values are collected in a vector $\boldsymbol{\lambda} \in \mathbb{R}_{+}^{r}$; and

- a displacement $u_{k}$ for each of the structural degrees of freedom $k=1, \ldots, n$. These values are collected in a vector $\boldsymbol{u} \in \mathbb{R}^{n}$.

The triple $(\boldsymbol{s}, \boldsymbol{\lambda}, \boldsymbol{u})$ will be referred to as state variables.

For a vector $\boldsymbol{v} \in \mathbb{R}^{q}$, and an index set $I=\left\{i_{1}, \ldots, i_{|I|}\right\} \subseteq\{1, \ldots, q\}$, we denote by $\boldsymbol{v}_{I}$ the subvector $\left(v_{i_{1}}, \ldots, v_{i_{|I|}}\right)^{t}$.

The values of the state variables for a specific design $\boldsymbol{x}$ are determined using various energy principles. Therefore, we define the complementary energy of the structure as

$$
\mathcal{E}(\boldsymbol{x}, \boldsymbol{s}, \boldsymbol{\lambda}):=\frac{1}{2} \sum_{i \in \mathcal{I}(\boldsymbol{x})} \frac{s_{i}^{2}}{E x_{i}}+\boldsymbol{g}^{t} \boldsymbol{\lambda},
$$

where $E$ is the Young modulus of the structural material, and $\boldsymbol{g} \in \mathbb{R}^{r}$ is a vector of gaps between the contact nodes and rigid obstacles. We also define the linearized strain energy:

$$
\Pi(\boldsymbol{x}, \boldsymbol{u}):=\frac{1}{2} \boldsymbol{u}^{t} \mathrm{~K}(\boldsymbol{x}) \boldsymbol{u},
$$

where $\mathrm{K}(\boldsymbol{x})$ is the stiffness matrix of the structure. The latter matrix is defined as

$$
\mathrm{K}(\boldsymbol{x}):=\sum_{i \in \mathcal{I}(\boldsymbol{x})} x_{i} \mathrm{~K}_{i}
$$

where $\mathrm{K}_{i}=E \mathrm{~B}_{i}^{t} \mathrm{~B}_{i}$ is the local stiffness matrix for the bar $i=1, \ldots, m$, and $\mathrm{B}_{i} \in \mathbb{R}^{1 \times n}$ is a kinematic transformation matrix for the bar $i=1, \ldots, m$.

In this notation the equilibrium state of the structure under the external load $f \in \mathbb{R}^{n}$ can be characterized using a primal-dual pair of convex quadratic programming problems:

$$
(\mathcal{C})_{\boldsymbol{x}}(\boldsymbol{f})\left\{\begin{array} { l } 
{ \operatorname { m i n } _ { ( \boldsymbol { s } , \boldsymbol { \lambda } ) } \mathcal { E } ( \boldsymbol { x } , \boldsymbol { s } , \boldsymbol { \lambda } ) , } \\
{ \text { s.t. } C ^ { t } \boldsymbol { \lambda } + \sum _ { i \in \mathcal { I } ( \boldsymbol { x } ) } \mathrm { B } _ { i } ^ { t } s _ { i } = \boldsymbol { f } , } \\
{ \boldsymbol { \lambda } \geq \mathbf { 0 } , }
\end{array} \quad ( \mathcal { P } ) _ { \boldsymbol { x } } ( \boldsymbol { f } ) \left\{\begin{array}{l}
\min _{\boldsymbol{u}} \Pi(\boldsymbol{x}, \boldsymbol{u})-\boldsymbol{f}^{t} \boldsymbol{u}, \\
\text { s.t. } \mathrm{C} \boldsymbol{u} \leq \boldsymbol{g},
\end{array}\right.\right.
$$

where $C \in \mathbb{R}^{r \times n}$ is a kinematic transformation matrix. We have implicitly assumed that the matrix $C$ is quasi-orthogonal, that is, that $\mathrm{CC}^{t}=\mathrm{I}$. The problem $(\mathcal{C})_{\boldsymbol{x}}(\boldsymbol{f})$ is known as the principle of minimum 
complementary energy, and the problem $(\mathcal{P})_{\boldsymbol{x}}(\boldsymbol{f})$ is the principle of minimum potential energy.

Equivalently, the equilibrium problem can be written as a KKT system for the pair $(\mathcal{C})_{\boldsymbol{x}}(\boldsymbol{f})$ and $(\mathcal{P})_{\boldsymbol{x}}(\boldsymbol{f})$. Define

$$
\mathrm{Q}(\boldsymbol{x}):=\left(\begin{array}{ccc}
\mathrm{B}^{t} & \mathrm{C}^{t} & 0 \\
0 & 0 & -\mathrm{C} \\
\mathrm{I} & 0 & -\mathrm{D}(\boldsymbol{x}) \mathrm{B}
\end{array}\right), \quad \boldsymbol{q}(\boldsymbol{f}):=\left(\begin{array}{c}
-\boldsymbol{f} \\
\boldsymbol{g} \\
\mathbf{0}
\end{array}\right),
$$

and $Y:=\mathbb{R}^{m} \times \mathbb{R}_{+}^{r} \times \mathbb{R}^{n}$, where $\mathrm{B} \in \mathbb{R}^{m \times n}$ is the matrix with rows $\mathrm{B}_{1}, \ldots, \mathrm{B}_{m}$, and $\mathrm{D}(\boldsymbol{x})=$ $E^{-1} \operatorname{diag}(\boldsymbol{x}) \in \mathbb{R}^{m \times m}$. Then, the pair $(\boldsymbol{s}, \boldsymbol{\lambda})$ solves $(\mathcal{C})_{\boldsymbol{x}}(\boldsymbol{f})$ and $\boldsymbol{u}$ solves $(\mathcal{P})_{\boldsymbol{x}}(\boldsymbol{f})$ if and only if the vector $\boldsymbol{y}^{*}=\left(\boldsymbol{s}^{t}, \boldsymbol{\lambda}^{t}, \boldsymbol{u}^{t}\right)^{t} \in Y$ solves the affine variational inequality problem $\operatorname{AVI}(\boldsymbol{q}(\boldsymbol{f}), \mathrm{Q}(\boldsymbol{x}), Y)$ [see, e.g., [FaP03] for the definition]:

$$
\left[Q(\boldsymbol{x}) \boldsymbol{y}^{*}+\boldsymbol{q}(\boldsymbol{f})\right]^{t}\left(\boldsymbol{y}-\boldsymbol{y}^{*}\right) \geq 0, \quad \text { for all } \boldsymbol{y} \in Y
$$

For the rest of the paper, we make the blanket assumption that for all positive designs $\boldsymbol{x}$ it holds that

$$
\operatorname{null} \mathrm{K}(\boldsymbol{x}) \cap \operatorname{rc}\left\{\boldsymbol{u} \in \mathbb{R}^{n} \mid \mathrm{C} \boldsymbol{u} \leq \boldsymbol{g}\right\}=\emptyset,
$$

where null( $(\cdot)$ denotes the null space of a given matrix, and $\mathrm{rc}(\cdot)$ is the recession cone of a given set (cf. [HKP99]). This assumption is necessary to in order to guarantee the feasibility of the topology optimization problems we are going to consider, and is weaker than assuming non-singularity of $\mathrm{K}(\boldsymbol{x})$ for positive designs (the latter assumption is "standard" for contact-less problems, and then essentially equivalent to (1) because the recession cone involved equals $\mathbb{R}^{n}$ in the contact-less case). The assumption (1) does not exclude problems where the structure is supported on rigid obstacles only (see Figure 3 ).

Either of the equilibrium problem formulations $(\mathcal{C})_{\boldsymbol{x}}(\boldsymbol{f}),(\mathcal{P})_{\boldsymbol{x}}(\boldsymbol{f})$, or $\operatorname{AVI}(\boldsymbol{q}(\boldsymbol{f}), \mathrm{Q}(\boldsymbol{x}), Y)$ has its advantages and disadvantages. For example, the problem $(\mathcal{C})_{\boldsymbol{x}}(\boldsymbol{f})$ possesses at most one optimal solution for every design $\boldsymbol{x} \in \mathbb{R}_{+}^{m}$; at the same time, the objective function $\mathcal{E}$ is only lower semicontinuous (and may be infinite) for some $\boldsymbol{x} \in \partial \mathbb{R}_{+}^{m}$ (cf. [PaP02]).

Our ultimate goal in this paper is to establish stationary conditions that must be verified by limit points of certain sequences of positive designs. We cannot use the equilibrium formulation given by the problem $(\mathcal{C})_{x}(f)$ for this purpose, because its objective function violates such a basic condition for sensitivity analysis as continuity. Neither is the problem $(\mathcal{P})_{\boldsymbol{x}}(\boldsymbol{f})$ suitable for us, because the design-to-state mapping it induces is not closed [PaP02]. Therefore, we will use the primal-dual characterization of the equilibrium given by $\operatorname{AVI}(\boldsymbol{q}(\boldsymbol{f}), \mathrm{Q}(\boldsymbol{x}), Y)$ in the sequel.

We close the subsection by defining the feasible set generated by the equilibrium constraint:

$$
\mathcal{F}(\boldsymbol{f}):=\left\{(\boldsymbol{x}, \boldsymbol{s}, \boldsymbol{\lambda}, \boldsymbol{u}) \subset \mathbb{R}_{+}^{m} \times \mathbb{R}^{m} \times \mathbb{R}_{+}^{r} \times \mathbb{R}^{n} \mid(\boldsymbol{s}, \boldsymbol{\lambda}, \boldsymbol{u}) \in \mathrm{SOL}(\boldsymbol{q}(\boldsymbol{f}), \mathrm{Q}(\boldsymbol{x}), Y)\right\} .
$$

\subsection{Weight minimization problem}

We use a stress constrained weight minimization problem of a truss subject to unilateral frictionless contact with some rigid obstacles as a representative of the difficult structural optimization problems. To skip one index and simplify the notation we consider a single load case only; this does not affect the applicability of our results to multiple load cases in any way.

The weight minimization problem can be written as follows:

$$
(\mathcal{W})\left\{\begin{array}{l}
\min _{(\boldsymbol{x}, \boldsymbol{s}, \boldsymbol{\lambda}, \boldsymbol{u})} w(\boldsymbol{x}):=\sum_{i=1}^{m} x_{i}, \\
\text { s.t. }(\boldsymbol{x}, \boldsymbol{s}, \boldsymbol{\lambda}, \boldsymbol{u}) \in \mathcal{F}(\boldsymbol{f}), \\
\quad \underline{\sigma}_{i} x_{i} \leq s_{i} \leq \bar{\sigma}_{i} x_{i}, \quad i=1, \ldots, m,
\end{array}\right.
$$

where $\underline{\sigma}_{i} \leq 0$ and $\bar{\sigma}_{i} \geq 0$ are the stress bounds in compression and tension for the bar $i=1, \ldots, m$, and $\mathcal{F}(\boldsymbol{f})$ is given by $(2)$.

The results of the present paper are of course applicable to a wider class of problems than $(\mathcal{W})$. For example, more general objective functions can be considered as long as they are reasonably regular [differentiable, or Lipschitz continuous w.r.t. $(\boldsymbol{x}, \boldsymbol{s}, \boldsymbol{\lambda}, \boldsymbol{u})$ ]; additional constraints may be considered (such as bounds on admissible displacements, local buckling constraints, or global stability constraints). However, to keep the notation simple we do not discuss such straightforward generalizations in detail.

\subsection{Singularity}

For illustrational purposes here we reproduce an academic example introduced in [Pet01]. 


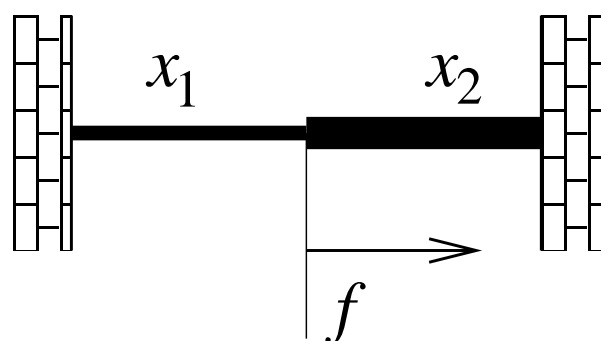

Figure 1: 1-bar truss structure.

Consider a simple 2-bar structure shown in Figure 1. Assume that $E=1, f=1,-\underline{\sigma}_{1}=\bar{\sigma}_{1}=1$, $-\underline{\sigma}_{2}=\bar{\sigma}_{2}=1 / 2$. In this case, the weight minimization problem is formulated as follows:

$$
\begin{array}{rlrl}
\underset{(\boldsymbol{x}, \boldsymbol{s}, \boldsymbol{\lambda}, \boldsymbol{u})}{\min } x_{1}+x_{2}, & \\
\text { s.t. } & x_{1} \geq 0, & x_{2} \geq 0, \\
-x_{1}+s_{1} \leq 0, & -x_{2}+s_{2} / 2 \leq 0, \\
-x_{1}-s_{1} \leq 0, & -x_{2}-s_{2} / 2 \leq 0, \\
s_{1}-x_{1} u=0, & s_{2}+x_{2} u=0, \\
s_{1}-s_{2}=1 . &
\end{array}
$$

The unique globally optimal solution to this problem is $\left(x_{1}^{*}, x_{2}^{*}, s_{1}^{*}, s_{2}^{*}, u\right)=(1,0,1,0,1)$. It is easy to verify that while the KKT conditions are satisfied at this point, MFCQ (hence, LICQ) is violated. This situation in structural optimization is rather typical, despite the fact that LICQ generically holds in nonlinear programming. For our developments it also means that, in the presence of contact conditions, we cannot assume that the resulting MPEC problem satisfies MPEC-LICQ, despite the corresponding generic result [Sch01]. (We also note that while weight minimization problems in a single-load case may be cast using linear programming techniques [StS04], we do not use this simplification since, arguably, single-load case problems rarely appear in practical situations.)

In what follows we will concentrate on constructing approximation problems that are (at least under some mild assumptions) qualified in the sense of Mangasarian and Fromowitz, and allow us to approximate both globally optimal solutions and stationary points of singular problems arising in truss topology optimization.

\section{Previous results}

\section{$2.1 \quad \varepsilon$-perturbation of Cheng and Guo and variations}

The so-called $\varepsilon$-perturbation of structural topology optimization problems, or approximation with a sequence of sizing optimization problems, has become a classic topic. Convergence results of this type allow one, at least in principle, to compute optimal solutions to structural topology optimization problems by solving a sequence of smooth non-convex approximating problems. Such approximations do not suffer from many numerical difficulties possessed by the original model problem $(\mathcal{W})$, so that efficient solvers are readily available.

For some truss topology optimization problems (such as, e.g., compliance minimization, possibly with so-called "strong" stress constraints [Ach98]) the naïve replacement of the lower bound 0 on design variables with a small positive value $\varepsilon>0$ tending to zero (whence the name- $\varepsilon$-perturbation) is sufficient. Such a strategy has been rigorously studied for trusses, without (Achtziger [Ach98]) and with (Patriksson and Petersson [PaP02]) unilateral constraints.

On the other hand, there are many other classes of topology optimization problems including important mechanical constraints (e.g., stress constraints [SvG68], local buckling constraints [GCY01], and global buckling constraints [Evg04]) where the simple strategy outlined above leads to erroneous results, owing to the complicated singular structure of the design domain near the points where the truss topology changes. Historically, the study of singularity phenomena for truss topology optimization problems started with problems including stress constraints only. Sved and Ginos [SvG68] observed that such problems may have 
singular solutions, and the properties of the feasible region were further investigated by Kirsch [Kir90], Cheng and Jiang [ChJ92], and Rozvany and Birker [RoB94]. Cheng and Guo [ChG97] were the first to propose a more sophisticated restriction-relaxation procedure, where not only lower bounds but also stress constraints were perturbed. They established the convergence of the optimal values of the perturbed problems to the optimal value of the original problem, while Petersson [Pet01] (using the continuity of certain design-to-state parameterized mappings) has established the convergence of optimal solutions. Since then, the $\varepsilon$-perturbation has been extended by many authors in many ways: Duysinx and Bendsøe [DuB98] and Duysinx and Sigmund [DuS98] considered continuum structures; Guo et al. [GCY01] included local buckling constraints into the problem; Patriksson and Petersson [PaP02] generalized the result for trusses including unilateral constraints; Evgrafov et al. [EPP03] considered the possibility of stochastic forces; and Evgrafov [Evg04] studied the linearized elastic stability constraint.

Despite the clear advantage of approximating the nonsmooth, singular optimization problem with a sequence of smooth and regular ones, all the sizing approximations considered above suffer from the same difficulty. While the underlying theoretical results are concerned with the approximation of the globally optimal solutions, in computational practice it is impossible to solve the non-convex approximating problems to global optimality. There are also negative results regarding this issue: the $\varepsilon$-perturbation approach may fail to find a globally optimal solution even for topology optimization problems with only 2 design variables (see $[\mathrm{StS} 01])$ !

The analysis of the convergence of stationary points to the approximating problems towards stationary points of the limiting (that is, original) problem is difficult; for example, the dependence of the equilibrium state of the structure upon the design near the points where the topology changes is nonsmooth, and even non-Lipschitz continuous.

In constructing a new $\varepsilon$-perturbation we try to address these issues, concentrating on the convergence of both globally optimal solutions and stationary points towards the respective limits.

\subsection{The extended formulation of Stolpe and Svanberg}

Recently, Stolpe and Svanberg [StS03] proposed an alternative method for the solution of the truss topology optimization problems including stress and local buckling constraints, which is based on the Karush-KuhnTucker (KKT) formulation of the equilibrium constraint. In this formulation the state variables are treated equally to the design variables, and artificial lower bounds on the design are unnecessary. In the absence of unilateral constraints, the formulation is suitable for any SQP algorithm, and for some numerical examples Stolpe and Svanberg report that such an algorithm has a better performance than an $\varepsilon$-perturbation based approach. Later, a branch-and-cut algorithm based on this formulation has been developed [Sto04]; furthermore, Achtziger [Ach03] has made the conjecture that every globally optimal solution to a topology optimization problem including stress and local buckling constraints (but not including unilateral constraints) is a KKT point in the extended formulation.

Unfortunately, the KKT formulation of the lower level equilibrium problem for trusses with unilateral constraints includes complementarity conditions, which are known to violate standard nonlinear programming constraint qualifications. Therefore, the extended formulation cannot be used directly to solve topology optimization problems for trusses in contact with rigid obstacles, or including tensile-only members (ropes or cables).

We therefore propose a new approximation scheme, which allows for the violation of the lower-level equilibrium conditions, and thus does not include the complicating complementarity constraints.

\section{Previous methods for general MPEC problems}

Among iterative algorithms for MPEC problems, our $\varepsilon$-perturbation method is special in that it combines relaxation (of the equilibrium conditions) and restrification (of the design space). Most iterative algorithms for general MPEC problems belong to the relaxation category, wherein constraints are penalized or complementarity conditions are smoothed. In the latter category, the methods of Facchinei et al. [FJQ99] and Scholtes [Sch01] have relations to ours that are interesting to explore, in order to analyze the strength of our convergence results. Due to the stronger regularity properties of the problems considered in [FJQ99, Sch01], their convergence results are shown to be stronger; we then seek to explain why local, iterative methods for our problem are unlikely to yield better convergence characteristics than those that we reach in this paper.

\subsection{The smoothing algorithm of [FJQ99]}

Consider the problem to 


$$
(\mathcal{M})\left\{\begin{array}{l}
\underset{(\boldsymbol{x}, \boldsymbol{y})}{\min } f(\boldsymbol{x}, \boldsymbol{y}), \\
\text { s.t. }\left\{\begin{array}{l}
\boldsymbol{x} \in X, \\
\boldsymbol{y} \text { solves } \operatorname{VI}(\boldsymbol{F}(\boldsymbol{x}, \cdot), C(\boldsymbol{x})),
\end{array}\right.
\end{array}\right.
$$

where $f: \mathbb{R}^{n+m} \mapsto \mathbb{R}$ is continuously differentiable, $X \subset \mathbb{R}^{n}$ is nonempty and compact, and, for each $\boldsymbol{x} \in X$ and for a continuously differentiable function $\boldsymbol{F}: \mathbb{R}^{n+m} \mapsto \mathbb{R}^{m}, \operatorname{VI}(\boldsymbol{F}(\boldsymbol{x}, \cdot), C(\boldsymbol{x}))$ denotes the variational inequality defined by the pair $(\boldsymbol{F}(\boldsymbol{x}, \cdot), C(\boldsymbol{x}))$,

$$
\boldsymbol{y} \in C(\boldsymbol{x}) ; \quad \boldsymbol{F}(\boldsymbol{x}, \boldsymbol{y})^{t}(\boldsymbol{z}-\boldsymbol{y}) \geq 0, \quad \boldsymbol{z} \in C(\boldsymbol{x}),
$$

where $C(\boldsymbol{x})=\left\{\boldsymbol{y} \in \mathbb{R}^{m} \mid g_{i}(\boldsymbol{x}, \boldsymbol{y}) \geq 0, i=1, \ldots, \ell\right\}, \boldsymbol{g}: \mathbb{R}^{n+m} \mapsto \mathbb{R}^{\ell}$ being twice continuously differentiable and concave in the second argument.

For the lower-level VI, we assume that $C(\boldsymbol{x}) \neq \emptyset$ for all $\boldsymbol{x}$ in an open set $A$ containing $X$, that $C(\boldsymbol{x})$ is uniformly compact on $A$ (with $C(\boldsymbol{x}) \subset B$ for some open bounded set $B \subset \mathbb{R}^{m}$ ), that $\boldsymbol{F}(\boldsymbol{x}, \cdot)$ is uniformly strongly monotone on $B$ for all $\boldsymbol{x} \in A$, and that for every pair $(\boldsymbol{x}, \boldsymbol{y})$ for which $\boldsymbol{x} \in X$ and $\boldsymbol{y}$ solves $\mathrm{VI}(\boldsymbol{F}(\boldsymbol{x}, \cdot), C(\boldsymbol{x}))$, the partial gradients $\nabla_{\boldsymbol{y}} g_{i}(\boldsymbol{x}, \boldsymbol{y}), i \in \mathcal{I}(\boldsymbol{x}, \boldsymbol{y}):=\left\{i=1, \ldots, \ell \mid g_{i}(\boldsymbol{x}, \boldsymbol{y})=0\right\}$, are linearly independent (that is, the linear independence CQ, LICQ).

By these assumptions, $\operatorname{VI}(\boldsymbol{F}(\boldsymbol{x}, \cdot), C(\boldsymbol{x}))$ has a unique solution for each $\boldsymbol{x} \in X$, and each lower-level VI is equivalent to the existence of a (unique) multiplier vector $\boldsymbol{\lambda} \in \mathbb{R}^{\ell}$ such that

$$
\begin{aligned}
\boldsymbol{F}(\boldsymbol{x}, \boldsymbol{y})-\nabla_{\boldsymbol{y}} \boldsymbol{g}(\boldsymbol{x}, \boldsymbol{y}) \boldsymbol{\lambda} & =\mathbf{0}^{m}, \\
\mathbf{0}^{\ell} \leq \boldsymbol{g}(\boldsymbol{x}, \boldsymbol{y}) \perp \boldsymbol{\lambda} & \geq \mathbf{0}^{\ell} .
\end{aligned}
$$

Let

$$
\boldsymbol{H}_{0}(\boldsymbol{x}, \boldsymbol{y}, \boldsymbol{z}, \boldsymbol{\lambda}):=\left(\begin{array}{c}
\boldsymbol{F}(\boldsymbol{x}, \boldsymbol{y})-\nabla_{\boldsymbol{y}} \boldsymbol{g}(\boldsymbol{x}, \boldsymbol{y}) \\
\boldsymbol{g}(\boldsymbol{x}, \boldsymbol{y})-\boldsymbol{z} \\
-2 \min (\boldsymbol{z}, \boldsymbol{\lambda})
\end{array}\right), \quad(\boldsymbol{x}, \boldsymbol{y}, \boldsymbol{z}, \boldsymbol{\lambda}) \in \mathbb{R}^{n+m+2 \ell} .
$$

The KKT system (3) is equivalent to the statement that $\boldsymbol{H}_{0}(\boldsymbol{x}, \boldsymbol{y}, \boldsymbol{z}, \boldsymbol{\lambda})=\mathbf{0}^{m+2 \ell}$. We therefore write

$$
(\mathcal{P})\left\{\begin{array}{l}
\min _{(\boldsymbol{x}, \boldsymbol{y})} f(\boldsymbol{x}, \boldsymbol{y}), \\
\text { s.t. }\left\{\begin{array}{l}
\boldsymbol{x} \in X, \\
\boldsymbol{H}_{0}(\boldsymbol{x}, \boldsymbol{y}, \boldsymbol{z}, \boldsymbol{\lambda})=\mathbf{0}^{m+2 \ell},
\end{array}\right.
\end{array}\right.
$$

which is an equivalent, non-smooth, restatement of $(\mathcal{M})$, in the sense that the two problems share global as well as local optimal solutions in $\boldsymbol{x}$ (cf. [FJQ99, Proposition 1]).

Facchinei et al. [FJQ99] consider a smooth reformulation of the problem $(\mathcal{P})$, as follows. We introduce the function $\phi: \mathbb{R}^{2} \mapsto \mathbb{R}$ by

$$
\phi_{\mu}(a, b):=\sqrt{(a-b)^{2}+4 \mu^{2}}-(a+b), \quad(a, b) \in \mathbb{R}^{2} .
$$

For this function, we have that ([FJQ99, Proposition 2])

$$
\phi_{\mu}(a, b)=0 \quad \Longleftrightarrow \quad a \geq 0, b \geq 0, a b=\mu^{2} .
$$

For $\mu=0, \phi_{\mu}(a, b)=-2 \min (a, b)$; for $\mu \neq 0, \phi_{\mu}$ is in $C^{\infty}$; and for every pair $(a, b), \lim _{\mu \rightarrow 0} \phi_{\mu}(a, b)=$ $-2 \min (a, b)$. The function $\phi_{\mu}$ therefore serves as a smooth perturbation of the min function. We consider replacing the operator $\boldsymbol{H}_{0}$ in the problem $(\mathcal{P})$ above with the smooth operator $\boldsymbol{H}_{\mu}$, defined by

$$
\boldsymbol{H}_{\mu}(\boldsymbol{x}, \boldsymbol{y}, \boldsymbol{z}, \boldsymbol{\lambda}):=\left(\begin{array}{c}
\boldsymbol{F}(\boldsymbol{x}, \boldsymbol{y})-\nabla_{\boldsymbol{y}} \boldsymbol{g}(\boldsymbol{x}, \boldsymbol{y}) \\
\boldsymbol{g}(\boldsymbol{x}, \boldsymbol{y})-\boldsymbol{z} \\
\boldsymbol{\Phi}_{\mu}(\boldsymbol{z}, \boldsymbol{\lambda})
\end{array}\right), \quad(\boldsymbol{x}, \boldsymbol{y}, \boldsymbol{z}, \boldsymbol{\lambda}) \in \mathbb{R}^{n+m+2 \ell},
$$

where $\boldsymbol{\Phi}(\boldsymbol{z}, \boldsymbol{\lambda}):=\left(\phi\left(z_{1}, \lambda_{1}\right), \ldots, \phi\left(z_{\ell}, \lambda_{\ell}\right)\right)$, thus defining the smoothing problem

$$
\left(\mathcal{P}_{\mu}\right)\left\{\begin{array}{l}
\min _{(\boldsymbol{x}, \boldsymbol{y})} f(\boldsymbol{x}, \boldsymbol{y}), \\
\text { s.t. }\left\{\begin{array}{l}
\boldsymbol{x} \in X, \\
\boldsymbol{H}_{\mu}(\boldsymbol{x}, \boldsymbol{y}, \boldsymbol{z}, \boldsymbol{\lambda})=\mathbf{0}^{m+2 \ell} .
\end{array}\right.
\end{array}\right.
$$


While $\left(\mathcal{P}_{0}\right)$ coincides with the non-smooth problem $(\mathcal{M})$, the problem $\left(\mathcal{P}_{\mu}\right)$ for $\mu \neq 0$ is a smooth optimization problem. We denote the feasible set to $\left(\mathcal{P}_{\mu}\right)$ by $\mathcal{F}_{\mu} \subset \mathbb{R}^{n+m+2 \ell}$. The function $\boldsymbol{H}_{\mu}$ is not only locally Lipschitz continuous for every $\mu$ but also regular (in the sense that the directional derivative exists in all directions and equals the Clarke derivative; cf. [FJQ99, Lemma 1]), and its generalized Jacobian with respect to $(\boldsymbol{y}, \boldsymbol{z}, \boldsymbol{\lambda})$ is non-singular for every $\mu$ and feasible point of the problem $\left(\mathcal{P}_{\mu}\right)$, cf. [FJQ99, Proposition 3]. Further, for every $\overline{\boldsymbol{x}} \in X$ and $\mu \in \mathbb{R}$ there exists a unique point in $\mathcal{F}_{\mu}$ such that its $\boldsymbol{x}$-part equals $\overline{\boldsymbol{x}}$, and this vector,

$$
\overline{\boldsymbol{w}}_{\mu}:=\left(\overline{\boldsymbol{x}}, \boldsymbol{y}_{\mu}(\overline{\boldsymbol{x}}), \boldsymbol{y}_{\mu}(\overline{\boldsymbol{x}}), \boldsymbol{\lambda}_{\mu}(\overline{\boldsymbol{x}})\right),
$$

is continuous in $\mu$. The feasible sets $\mathcal{F}_{\mu}$ of the problem $\left(\mathcal{P}_{\mu}\right)$ are non-empty and uniformly compact, whence the problems $\left(\mathcal{P}_{\mu}\right)$ have optimal solutions.

The first-order optimality conditions for the problem $\left(\mathcal{P}_{\mu}\right)$ can be written as follows: with $\boldsymbol{L}(\boldsymbol{x}, \boldsymbol{y}, \boldsymbol{\lambda}):=$ $\boldsymbol{F}(\boldsymbol{x}, \boldsymbol{y})-\nabla_{\boldsymbol{y}} \boldsymbol{g}(\boldsymbol{x}, \boldsymbol{y}) \boldsymbol{\lambda}$, if $(\boldsymbol{x}, \boldsymbol{y}, \boldsymbol{z}, \boldsymbol{\lambda})$ is a locally optimal solution to $\left(\mathcal{P}_{\mu}\right)$, then there exist vectors $(\boldsymbol{\theta}, \boldsymbol{\rho}, \boldsymbol{\sigma}) \in$ $\mathbb{R}^{m+2 \ell}$ and $s \in N_{X}(\boldsymbol{x}) \times \mathbb{R}^{m+2 \ell}$ such that (cf. [FJQ99, Theorem 2])

$$
\mathbf{0}^{n+m} \in \nabla f(\boldsymbol{x}, \boldsymbol{y})+\nabla \boldsymbol{L}(\boldsymbol{x}, \boldsymbol{y}, \boldsymbol{\lambda}) \boldsymbol{\theta}+\nabla(\boldsymbol{g}(\boldsymbol{x}, \boldsymbol{y})-\boldsymbol{z}) \boldsymbol{\rho}+\sum_{i=1}^{\ell} \partial \phi_{\mu}\left(z_{i}, \lambda_{i}\right) \sigma_{i}+M\|(1, \boldsymbol{\theta}, \boldsymbol{\rho}, \boldsymbol{\sigma})\| \boldsymbol{s},
$$

where $M$ is a Lipschitz constant for $\left(f, \boldsymbol{H}_{\mu}\right)$ around $(\boldsymbol{x}, \boldsymbol{y}, \boldsymbol{z}, \boldsymbol{\lambda})$. Since the multiplier for $\nabla f(\boldsymbol{x}, \boldsymbol{y})$ is non-zero (it then equals 1 , without any loss of generality), this condition is stronger than the Fritz-John conditions, and is in fact the KKT conditions for the problem. While one may then refer to this condition for $\mu=0$ as the KKT conditions for the MPEC problem $(\mathcal{M})$, Facchinei et al. [FJQ99] refer to it is as strong C-stationarity (SCS).

A global version of the smoothing algorithm is immediate. A more practical algorithm is obtained by replacing, in the algorithm above, global optimality in $\left(\mathcal{P}_{\mu_{\tau}}\right)$ of the vector $\boldsymbol{w}^{\tau}$ by stationarity in the sense of the KKT system (5). For this algorithm, it is shown in [FJQ99, Theorem 4] that the sequence $\left\{\boldsymbol{w}^{\tau}\right\}$ of KKT points in $\left(\mathcal{P}_{\mu_{\tau}}\right)$ is bounded and every limit point is an SCS point in $(\mathcal{P})$. A crucial part of the proof is the continuity property of any sequence of KKT points in the problem $\left(\mathcal{P}_{\mu}\right)$ as $\mu$ tends to zero (cf. [FJQ99, Proposition 4]). The proof of the convergence result also establishes the important result that the sequence $\left\{\left(\boldsymbol{\theta}^{\tau}, \boldsymbol{\rho}^{\tau}, \boldsymbol{\sigma}^{\tau}\right)\right\}$ of KKT multipliers is bounded. This is a crucial part of any analysis of the stationarity property of a limit point.

A yet more practical algorithm is also devised, in which the sequence $\left\{\boldsymbol{w}^{\tau}\right\}$ of vectors is allowed to be defined by near-feasible and approximate KKT points. In other words, in each iteration $\tau$, the distance from the vector $\boldsymbol{w}^{\tau}$ to the feasible set $\mathcal{F}_{\mu}$ of the problem $\left(\mathcal{P}_{\mu_{\tau}}\right)$ is bounded by $\varepsilon_{\tau}>0$, and the Euclidean length of the vector defining the right-hand side of the inclusion (5) is also bounded above by this value. Theorem 5 in [FJQ99] then states that if $\left\{\varepsilon_{\tau}\right\} \downarrow 0$ as $\left\{\mu_{\tau}\right\} \rightarrow 0$, then the sequence $\left\{\boldsymbol{w}^{\tau}\right\}$ of approximate KKT points is bounded and every limit point is, again, a SCS point in $(\mathcal{P})$.

The latter algorithm was coded and tested in [FJQ99] on some small and medium-size MPEC problems; each problem $\left(\mathcal{P}_{\mu_{\tau}}\right)$ was then solved by utilizing an SQP algorithm. They report that it compares favourably with, for example, the implicit programming algorithms proposed in [Out94, OuZ95].

\subsection{The regularization algorithm in [Sch01]}

Scholtes [Sch01] considers the parameterized problem to

$$
(\mathcal{N} \mathcal{L} \mathcal{P}(t))\left\{\begin{aligned}
\min _{\boldsymbol{x}} f(\boldsymbol{x}), & \\
\text { s.t. } & \boldsymbol{g}(\boldsymbol{x}) \\
\boldsymbol{h}(\boldsymbol{x}) & \leq \mathbf{0}^{p}, \\
\boldsymbol{G}(\boldsymbol{x}) & \geq \mathbf{0}^{q}, \\
\boldsymbol{H}(\boldsymbol{x}) & \geq \mathbf{0}^{m}, \\
G_{i}(\boldsymbol{x}) H_{i}(\boldsymbol{x}) & \leq t,
\end{aligned}\right.
$$

where $f, \boldsymbol{g}, \boldsymbol{h}, \boldsymbol{G}$, and $\boldsymbol{H}$ are continuously differentiable. The original problem, $(\mathcal{N} \mathcal{L P}(0))$, is ill-posed, whence it is natural to consider solving $(\mathcal{N} \mathcal{L P}(t))$ for $t \downarrow 0$. An MPEC-LICQ is introduced by which, disregarding the (relaxed) complementarity conditions, the active constraints are all linearly independent. Three stationarity conditions are introduced, which, in addition to the weak stationarity condition

$$
\nabla f(\overline{\boldsymbol{x}})+\sum_{i \in \mathcal{I}_{g}(\overline{\boldsymbol{x}})} \bar{\lambda}_{i} \nabla g_{i}(\overline{\boldsymbol{x}})+\sum_{j \in \mathcal{I}_{h}(\bar{x})} \bar{\mu}_{j} \nabla h_{j}(\overline{\boldsymbol{x}})-\sum_{k \in \mathcal{I}_{G}(\bar{x})} \bar{\gamma}_{k} \nabla G_{k}(\overline{\boldsymbol{x}})-\sum_{\ell \in \mathcal{I}_{H}(\bar{x})} \bar{\nu}_{\ell} \nabla H_{\ell}(\overline{\boldsymbol{x}})=\mathbf{0}^{n},
$$


impose the following additional conditions on the complementarity part:

- $C$-stationarity: $m \in I_{G}(\overline{\boldsymbol{x}}) \cap I_{H}(\overline{\boldsymbol{x}})$ implies that $\bar{\gamma}_{m} \bar{\nu}_{m} \geq 0$;

- M-stationarity: all $m \in I_{G}(\overline{\boldsymbol{x}}) \cap I_{H}(\overline{\boldsymbol{x}})$ satisfy either $\bar{\gamma}_{m} \bar{\nu}_{m}>0$ or $\bar{\gamma}_{m} \bar{\nu}_{m}=0$;

- strong stationarity: $m \in I_{G}(\overline{\boldsymbol{x}}) \cap I_{H}(\overline{\boldsymbol{x}})$ implies that $\bar{\gamma}_{m} \geq 0$ and $\bar{\nu}_{m} \geq 0$.

The latter is, under MPEC-LICQ, equivalent to a B-stationarity condition introduced by the author. Based on these conditions, Scholtes establishes that for an arbitrary sequence $\left\{t_{\tau}\right\} \downarrow 0$ and a corresponding sequence $\left\{\boldsymbol{x}_{\tau}\right\}$ of stationary points to $\left(\mathcal{N} \mathcal{L} \mathcal{P}\left(t_{\tau}\right)\right.$ ), a limit point $\overline{\boldsymbol{x}}$ of $\left\{\boldsymbol{x}_{\tau}\right\}$ satisfies the following ([Sch01, Theorems 3.1 and 3.3]):

- $\bar{x}$ is C-stationary;

- $\overline{\boldsymbol{x}}$ is B-stationary if and only if $\bar{\gamma}_{m}=\bar{\nu}_{m}=0$ for every $m \in I_{G}(\overline{\boldsymbol{x}}) \cap I_{H}(\overline{\boldsymbol{x}}) \cap I_{0}$, where

$$
I_{0}:=\left\{m \mid m \in I_{G H}\left(\boldsymbol{x}_{\tau}, t_{\tau}\right) \text { for infinitely many } \tau\right\} ;
$$

- if a second-order sufficiency condition holds at each $\boldsymbol{x}_{\tau}$ then $\overline{\boldsymbol{x}}$ is M-stationary.

Randomly generated problems with $f$ quadratic and the constraint functions all being affine were tested against a smoothing type method wherein the last constraint in $(\mathcal{N} \mathcal{L} \mathcal{P}(t))$ was replaced by the corresponding equality constraint.

Solving 100 problems, all 100 problems were successful in the sense that the last problem (with $t=10^{-16}$; the starting value was always unity) was solvable. (The solver for each problem was based on Matlab:s fmincon function.) In the case of the smoothing algorithm, 85 problems were successful, and in 66 cases the solver encountered solvability problems somewhere between the initial and terminal value of $t$. The smoothing algorithm was not only less robust but also produced worse solutions in some cases: in 47 of the 85 problems mentioned above, the two methods produced the same solution, while in the 36 cases the regularization method produced better solutions (at most $12 \%$ better).

Scholtes conclusion is that regularization by means of relaxation, as in the construction of $(\mathcal{N} \mathcal{L P}(t))$ is a sensible way of stabilizing existing NLP codes when extended to MPEC problems. He also remarks that thanks to the fact that problems constructed and solved in a relaxation/regularization method encompasses the original feasible set, while smoothing methods do not, which may imply the possibility to faster encounter active sets among the complementarity conditions.

\section{A new smoothing approach to topology optimization}

\subsection{Motivation}

The smoothing algorithm described in Section 3 may unfortunately not be applied to truss topology optimization problems, out of which $(\mathcal{W})$ is a typical example. The latter problem violates several assumptions that are vital for the smoothing algorithm of Facchinei et al. [FJQ99], the most important being the lack of the uniform strong monotonicity by the lower-level problem $\operatorname{AVI}(\boldsymbol{q}(\boldsymbol{f}), \mathrm{Q}(\boldsymbol{x}), Y)$. In addition, some of the variables (that is, $\boldsymbol{u}$ ) may not be uniformly bounded, and upper-level joint constraints (such as stress constraints) are essential in the problem $(\mathcal{W})$.

In order to overcome the difficulties outlined we introduce an alternative perturbation scheme for solving stress constrained weight minimization problems for trusses including unilateral constraints. It resembles the $\varepsilon$-perturbation approach of Cheng and Guo [ChG97] (cf. Section 2.1) by the fact that we introduce positive lower bounds on the design variables, thus restricting the design domain. There are important differences, however: instead of relaxing the technological constraints (e.g., stress constraints in the original paper [ChG97]) we relax the equilibrium constraint; to accomplish this, we formulate the optimization problem using both the design and the state variables, similarly to the extended formulation of Stolpe and Svanberg [StS03] (cf. Section 2.2). 


\subsection{Relaxed equilibrium problem}

Formally, fix an arbitrary $\varepsilon \geq 0$ and consider the following perturbation of the feasible set $\mathcal{F}(\boldsymbol{f})(\mathrm{cf}$. $(2))$ :

$$
\begin{aligned}
\mathcal{F}^{\varepsilon}(\boldsymbol{f}):=\left\{(\boldsymbol{x}, \boldsymbol{s}, \boldsymbol{\lambda}, \boldsymbol{u}) \in \mathbb{R}_{+}^{m} \times \mathbb{R}^{m} \times \mathbb{R}_{+}^{r} \times \mathbb{R}^{n} \mid \boldsymbol{x}\right. & \geq o(\varepsilon) \mathbf{1}^{m}, \\
\mathcal{E}(\boldsymbol{x}, \boldsymbol{s}, \boldsymbol{\lambda})+\Pi(\boldsymbol{x}, \boldsymbol{u})-\boldsymbol{f}^{t} \boldsymbol{u} & \leq \varepsilon \\
\mathrm{B}^{t} \boldsymbol{s}+\mathrm{C}^{t} \boldsymbol{\lambda} & =\boldsymbol{f}, \\
\mathrm{C} \boldsymbol{u} & \leq \boldsymbol{g}\},
\end{aligned}
$$

where $o(\varepsilon)$ is a positive function of $\varepsilon$ such that $\lim _{\varepsilon \downarrow 0} o(\varepsilon) / \varepsilon=0$. Of course, the weak duality theorem for convex problems implies that $\mathcal{F}^{0}(\boldsymbol{f})=\mathcal{F}(\boldsymbol{f})$; for positive values of $\varepsilon$ the "state" variables $(\boldsymbol{s}, \boldsymbol{\lambda}, \boldsymbol{u})$ (which in the extended formulation play a role equal to that of the design variables $\boldsymbol{x}$, and do not correspond to an equilibrium state of the truss anymore) are required to be primal-dual feasible, but only $\varepsilon$-optimal.

Finally, for every $\varepsilon>0$ we consider the following perturbed version of the stress constrained weight minimization problem:

$$
\left(\mathcal{W}^{\varepsilon}\right)\left\{\begin{array}{l}
\min _{(\boldsymbol{x}, \boldsymbol{s}, \boldsymbol{\lambda}, \boldsymbol{u})} w(\boldsymbol{x}), \\
\text { s.t. }(\boldsymbol{x}, \boldsymbol{s}, \boldsymbol{\lambda}, \boldsymbol{u}) \in \mathcal{F}^{\varepsilon}(\boldsymbol{f}), \\
\quad \underline{\sigma}_{i} x_{i} \leq s_{i} \leq \bar{\sigma}_{i} x_{i}, \quad i=1, \ldots, m .
\end{array}\right.
$$

From the theoretical point of view, allowing for $\varepsilon$-optimal solutions to the lower-level equilibrium problem means that we "regularize" the bi-level programming problem $(\mathcal{W})$, in the sense defined by [LiM97]; this will allow us to obtain the convergence of both globally optimal solutions and stationary points (see below).

At least of equal importance is the following practical interpretation of the method. If we apply typical "standard" non-linear programming methods to the original non-relaxed problem $(\mathcal{W})$, in many cases it is reasonable to assume that the underlying numerical method does in fact solve somewhat relaxed problem. For example, in methods based on implicit programming, we typically solve the equilibrium problem only approximately (especially for designs $\boldsymbol{x}$ close to the boundary of $\mathbb{R}_{+}^{m}$, when the stiffness matrix $\mathrm{K}(\boldsymbol{x})$ becomes ill-conditioned); at the same time it is easy to keep (linear) feasibility of the state variables in the equilibrium problem. Even if we do not use implicit programming and treat all variables involved in $(\mathcal{W})$ as independent, sometimes it is reasonable to require that the linear constraints [primal-dual feasibility of $(\boldsymbol{s}, \boldsymbol{\lambda}, \boldsymbol{u})$ and stress constraints] are satisfied with higher accuracy than the non-linear constraints expressing optimality of the state, which again results in a feasible set similar to $\mathcal{F}^{\varepsilon}(\boldsymbol{f})$. In particular, this may explain the success of SNOPT in solving the weight minimization problem with stress and local buckling constraints reported in [StS03].

Remark 4.1. In the multiple-load case, the problem $\left(\mathcal{W}^{\varepsilon}\right)$ will have several constraints of the form $\left(\boldsymbol{x}, \boldsymbol{s}^{k}, \boldsymbol{\lambda}^{k}, \boldsymbol{u}^{k}\right) \in \mathcal{F}^{\varepsilon}\left(\boldsymbol{f}^{k}\right)$, where $\boldsymbol{f}^{k}$ is a vector of external forces corresponding to the load case $k$, and the triple $\left(\boldsymbol{s}^{k}, \boldsymbol{\lambda}^{k}, \boldsymbol{u}^{k}\right) \in \mathbb{R}^{m} \times \mathbb{R}_{+}^{r} \times \mathbb{R}^{n}$ represents the "state" variables for the load case $k, k=1, \ldots, \ell$.

In the rest of the section, we study the theoretical properties of the point-to-set mapping $\varepsilon \rightrightarrows \mathcal{F}^{\varepsilon}(\boldsymbol{f})$ which will allow us to establish the convergence of globally optimal solutions as well as stationary points as $\varepsilon \downarrow 0$.

\subsection{Properties of $\varepsilon \rightrightarrows \mathcal{F}^{\varepsilon}(f)$}

In this section we show that the point-to-set mapping $\varepsilon \rightrightarrows \mathcal{F}^{\varepsilon}(\boldsymbol{f})$ enjoys most of the nice properties one can expect from a point-to-set mapping: under some mild conditions it has compact (although, unfortunately, non-convex) images, and is closed and lower semicontinuous at zero [AuF90, Chapter 1]. Furthermore, in Proposition 4.6 we demonstrate the continuity of the design-to-force "sub-mapping" $\boldsymbol{x} \rightrightarrows(\boldsymbol{s}, \boldsymbol{\lambda})$ (see Proposition 4.6 for the formal definition), a property originally established for the unperturbed feasible set $\mathcal{F}$ by Petersson [Pet01] for trusses without unilateral constraints, and later generalized by Patriksson and Petersson [PaP02].

We formulate the results as a sequence of short propositions.

Proposition 4.2 (Closed images). For each $\varepsilon \geq 0$ the set $\mathcal{F}^{\varepsilon}(\boldsymbol{f})$ is closed.

Proof. The claim follows easily from the lower semicontinuity of $\mathcal{E}(\cdot, \cdot, \cdot)$ (cf. [PaP02, Lemma 3.2]) together with the continuity of the other functions defining $\mathcal{F}^{\varepsilon}(\boldsymbol{f}), \varepsilon \geq 0$.

Proposition 4.3 (Lower semicontinuity). The multi-function $\varepsilon \rightrightarrows \mathcal{F}^{\varepsilon}(\boldsymbol{f})$ is lower semicontinuous at zero. 
Proof. Let $(\boldsymbol{x}, \boldsymbol{s}, \boldsymbol{\lambda}, \boldsymbol{u}) \in \mathcal{F}(\boldsymbol{f})$. Then, $\left\{\left(\boldsymbol{x}+o(\varepsilon) \mathbf{1}^{m}, \boldsymbol{s}, \boldsymbol{\lambda}, \boldsymbol{u}\right)\right\} \in \mathcal{F}^{\varepsilon}(\boldsymbol{f})$ for all enough small $\varepsilon>0$, where $\mathbf{1}^{m}=(1, \ldots, 1)^{t} \in \mathbb{R}_{+}^{m}$.

Remark 4.4. The same construction establishes the lower semicontinuity of the multi-functions $\varepsilon \rightarrow\left(\mathcal{F}^{\varepsilon} \cap\right.$ $K$ ), where (independent of $\varepsilon$ ) the closed set $K$ may represent stress, stiffness, or global stability constraints, or any combination thereof.

We stress that the classic $\varepsilon$-perturbation of Cheng and Guo [ChG97] results in a 1.s.c. mapping including design variables only; i.e., there might be some displacement vectors corresponding to the limiting design that cannot be approximated with the displacements corresponding to positive designs.

Proposition 4.5 (Closedness). The multi-function $\varepsilon \rightrightarrows \mathcal{F}^{\varepsilon}(f)$ is closed at zero.

Proof. The claim follows from the lower semicontinuity of $\mathcal{E}(\cdot, \cdot, \cdot)$ ( $\mathrm{cf}$. [PaP02, Lemma 3.2]) together with the continuity of the other functions, defining the sets $\mathcal{F}^{\varepsilon}(\boldsymbol{f}), \varepsilon \geq 0$.

Proposition 4.6 (Continuity of the design-to-force mapping). Let $\left\{\varepsilon_{k}\right\}$ be a positive sequence, converging to zero. Assume that $\left(\boldsymbol{x}^{k}, \boldsymbol{s}^{k}, \boldsymbol{\lambda}^{k}, \boldsymbol{u}^{k}\right) \in \mathcal{F}^{\varepsilon_{k}}(\boldsymbol{f})$, and that $\left\{\boldsymbol{x}^{k}\right\} \rightarrow \boldsymbol{x}$. Suppose further that for each $k=1,2, \ldots, i=1, \ldots, m$, the stress constraints $\underline{\sigma}_{i} x_{i}^{k} \leq s_{i}^{k} \leq \bar{\sigma}_{i} x_{i}^{k}$ constraints are satisfied. Then, $\left\{\left(\boldsymbol{s}^{k}, \boldsymbol{\lambda}^{k}\right)\right\} \rightarrow(\boldsymbol{s}, \boldsymbol{\lambda})$, this limit vector solves $(\mathcal{C})_{\boldsymbol{x}}(\boldsymbol{f})$, and there is a vector $\boldsymbol{u}$ solving $(\mathcal{P})_{\boldsymbol{x}}(\boldsymbol{f})$. [In particular, $(\boldsymbol{x}, \boldsymbol{s}, \boldsymbol{\lambda}, \boldsymbol{u}) \in \mathcal{F}$.]

Proof. The additional stress constraints imply the uniform boundedness of the sequence of complementary energies $\left\{\mathcal{E}\left(\boldsymbol{x}^{k}, \boldsymbol{s}^{k}, \boldsymbol{\lambda}^{k}\right)\right\}$, as has been established in [PaP02]. Therefore, the sequence $\left\{\left(\boldsymbol{s}^{k}, \boldsymbol{\lambda}^{k}\right)\right\}$ is bounded, owing to the coercivity of $\mathcal{E}$, which is locally uniform with respect to the design. Let $(\boldsymbol{s}, \boldsymbol{\lambda})$ be an arbitrary limit point of this sequence. The lower semicontinuity of $\mathcal{E}$ and the uniform boundedness of energies yield that

$$
\mathcal{E}(\boldsymbol{x}, \boldsymbol{s}, \boldsymbol{\lambda}) \leq \liminf _{k \rightarrow \infty} \mathcal{E}\left(\boldsymbol{x}^{k}, \boldsymbol{s}^{k}, \boldsymbol{\lambda}^{k}\right)<\infty .
$$

Therefore, the problem $(\mathcal{C})_{\boldsymbol{x}}(\boldsymbol{f})$ is feasible and thus possesses a unique optimal solution (cf. [PaP02, Theorem 2.1]).

Let now $(\tilde{\boldsymbol{s}}, \tilde{\boldsymbol{\lambda}})$ be an arbitrary force distribution that is feasible in $(\mathcal{C})_{\boldsymbol{x}}(\boldsymbol{f})$. Then, from the $\varepsilon_{k^{-}}$-optimality of $\left(\boldsymbol{s}^{k}, \boldsymbol{\lambda}^{k}\right)$ and feasibility of $(\tilde{\boldsymbol{s}}, \tilde{\boldsymbol{\lambda}})$ in $(\mathcal{C})_{\boldsymbol{x}^{k}}(\boldsymbol{f})$ it follows that

$$
\mathcal{E}(\boldsymbol{x}, \boldsymbol{s}, \boldsymbol{\lambda}) \leq \liminf _{k \rightarrow \infty} \mathcal{E}\left(\boldsymbol{x}^{k}, \boldsymbol{s}^{k}, \boldsymbol{\lambda}^{k}\right) \leq \lim _{k \rightarrow \infty} \mathcal{E}\left(\boldsymbol{x}^{k}, \tilde{\boldsymbol{s}}, \tilde{\boldsymbol{\lambda}}\right)+\varepsilon_{k}=\mathcal{E}(\boldsymbol{x}, \tilde{\boldsymbol{s}}, \tilde{\boldsymbol{\lambda}}),
$$

where the equality follows from the continuity of $\mathcal{E}(\cdot, \tilde{\boldsymbol{s}}, \tilde{\boldsymbol{\lambda}})$ (cf. [PaP02, Lemma 3.2]). Therefore, $(\boldsymbol{s}, \boldsymbol{\lambda})$ is optimal in $(\mathcal{C})_{\boldsymbol{x}}(\boldsymbol{f})$. It follows that $(\boldsymbol{s}, \boldsymbol{\lambda})$ must be the only limit point of the sequence $\left\{\left(\boldsymbol{s}^{k}, \boldsymbol{\lambda}^{k}\right)\right\}$.

The existence of at least one dual optimal solution $\boldsymbol{u}$ to $(\mathcal{P})_{\boldsymbol{x}}(\boldsymbol{f})$ follows.

Proposition 4.7 (Compact images). For every $\varepsilon>0$ and every constant $M>0$ the set $\{(\boldsymbol{x}, \boldsymbol{s}, \boldsymbol{\lambda}, \boldsymbol{u}) \in$ $\left.\mathcal{F}^{\varepsilon} \mid\|\boldsymbol{x}\| \leq M\right\}$ is compact.

Proof. The function $\mathcal{E}(\boldsymbol{x}, \boldsymbol{s}, \boldsymbol{\lambda})+\Pi(\boldsymbol{x}, \boldsymbol{u})-\boldsymbol{f}^{t} \boldsymbol{u}$ is continuous as well as coercive in $(\boldsymbol{s}, \boldsymbol{\lambda}, \boldsymbol{u})$, uniformly in $\boldsymbol{x}$ for all $\boldsymbol{x} \geq o(\varepsilon) \mathbf{1}^{m}$, with $\|\boldsymbol{x}\| \leq M$.

In the subsections that follow we apply the continuity results we have just established to show that the $\varepsilon$-perturbed problems can indeed be used as approximating problems for small $\varepsilon$, both if we are interested in globally optimal solutions and stationary points.

\subsection{Regularity of $\left(\mathcal{W}^{\varepsilon}\right)$}

To be of practical use, every approximating problem $\left(\mathcal{W}^{\varepsilon}\right)$ should be easier to solve than the original problem $(\mathcal{W})$. Clearly, the functions defining the constraints of $\left(\mathcal{W}^{\varepsilon}\right)$ are continuously differentiable on some neighbourhood of the feasible set $\mathcal{F}^{\varepsilon}$ for every $\varepsilon>0$; therefore, the smooth Fritz-John conditions must hold at optimal points. The following (purely academic) example shows that the feasible sets of the optimization problems $\left(\mathcal{W}^{\varepsilon}\right)$ do not in general verify MFCQ, and therefore we cannot expect the KKT conditions to be satisfied at every point of local minimum. On the other hand, in Proposition 4.9 we show that under rather mild additional conditions MFCQ is verified, so that standard nonlinear programming algorithms can be used to find locally optimal solutions of $\left(\mathcal{W}^{\varepsilon}\right)$. 


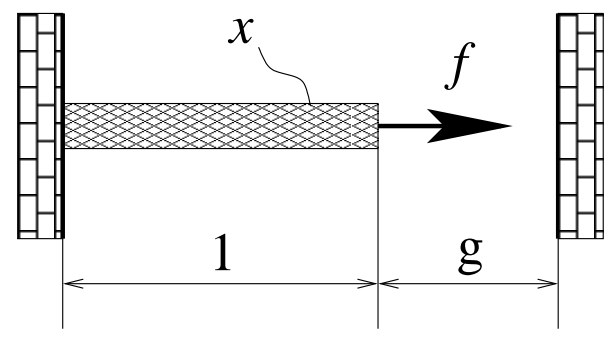

Figure 2: 1-bar truss structure.

Example 4.8. Consider the simple 1-bar structure shown in Figure 2 that is made of (academic) material with the Young modulus $E=1$. Let $f=3, g=2, \varepsilon=1, \bar{\sigma}=(2-\sqrt{2})$, and consider the point of global minimum $(x, s, \lambda, u)=(1,2-\sqrt{2}, 1+\sqrt{2}, 2)$. At this feasible point in $\left(\mathcal{W}^{\varepsilon}\right)$ the active constraints are:

$$
\left\{\begin{array} { l } 
{ s + \lambda = f , } \\
{ - x \leq - \varepsilon ^ { 2 } , } \\
{ u \leq g , } \\
{ s \leq \overline { \sigma } x , } \\
{ \frac { s ^ { 2 } } { 2 x } + g \lambda + \frac { 1 } { 2 } u ^ { 2 } x - f u \leq \varepsilon }
\end{array} \Leftrightarrow \left\{\begin{array}{l}
s+\lambda=3, \\
-x \leq-1, \\
u \leq 2, \\
s \leq(2-\sqrt{2}) x, \\
\frac{s^{2}}{2 x}+2 \lambda+\frac{1}{2} u^{2} x-3 u \leq 1 .
\end{array}\right.\right.
$$

It is easy to verify that there is no direction $\boldsymbol{d} \in \mathbb{R}^{4}$ such that

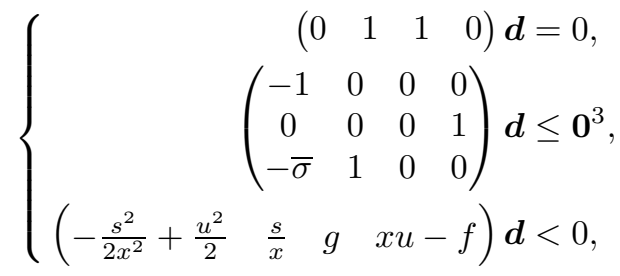

so that MFCQ is violated at $(x, s, \lambda, u)$.

While MFCQ is violated at the point of global minimum in the Example 4.8, this does not prevent the KKT conditions to hold at this point, because the more basic Abadie's CQ is still verified. While for realistic trusses the latter $\mathrm{CQ}$ is close to impossible to verify, the following result resolves the problem of verifying a $\mathrm{CQ}$ in most practical situations.

Proposition 4.9. Let $(\boldsymbol{x}, \boldsymbol{s}, \boldsymbol{\lambda}, \boldsymbol{u})$ be a point of local minimum for $\left(\mathcal{W}^{\varepsilon}\right), \varepsilon>0$. Suppose that any of the following conditions are verified:

(i) $\mathcal{E}(\boldsymbol{x}, \boldsymbol{s}, \boldsymbol{\lambda})+\Pi(\boldsymbol{x}, \boldsymbol{u})-\boldsymbol{f}^{t} \boldsymbol{u}<\varepsilon$;

(ii) $r=0$, that is, no rigid obstacles are present;

(iii) $\boldsymbol{u}$ is not the equilibrium displacement corresponding to $\boldsymbol{x}$.

Then, Abadie's $C Q$ hold at $(\boldsymbol{x}, \boldsymbol{s}, \boldsymbol{\lambda}, \boldsymbol{u})$. In particular, the KKT-conditions for $\left(\mathcal{W}^{\varepsilon}\right)$ hold at this point.

Proof. Suppose that $(i)$ holds. Then the relaxed equilibrium constraint is passive, and the feasible set of the problem $\left(\mathcal{W}^{\varepsilon}\right)$ is locally around $(\boldsymbol{x}, \boldsymbol{s}, \boldsymbol{\lambda}, \boldsymbol{u})$ defined by affine constraints only, which guarantees Abadie's CQ.

Alternatively, assume that there are no rigid obstacles, i.e., (ii) holds. Consider the direction $\boldsymbol{d}=$ $\left(\alpha \boldsymbol{x}, \mathbf{0}^{m}, \mathbf{0}^{r},-\beta \boldsymbol{u}\right)$, where $\alpha>0, \beta \geq 0$ are parameters to be determined. This direction is feasible with respect to all linear constraints of $\left(\mathcal{W}^{\varepsilon}\right)$. Furthermore, an easy calculation shows that

$$
\nabla\left[\mathcal{E}(\boldsymbol{x}, \boldsymbol{s}, \boldsymbol{\lambda})-\Pi(\boldsymbol{x}, \boldsymbol{u})-\boldsymbol{f}^{t} \boldsymbol{u}\right]^{t} \boldsymbol{d}=-\alpha[\mathcal{E}(\boldsymbol{x}, \boldsymbol{s}, \boldsymbol{\lambda})-\Pi(\boldsymbol{x}, \boldsymbol{u})]-\beta\left[2 \Pi(\boldsymbol{x}, \boldsymbol{u})-\boldsymbol{f}^{t} \boldsymbol{u}\right]<0,
$$

for some $\alpha>0, \beta \geq 0$, owing to the inequality

$$
0<\varepsilon=[\mathcal{E}(\boldsymbol{x}, \boldsymbol{s}, \boldsymbol{\lambda})-\Pi(\boldsymbol{x}, \boldsymbol{u})]+\left[2 \Pi(\boldsymbol{x}, \boldsymbol{u})-\boldsymbol{f}^{t} \boldsymbol{u}\right] .
$$

Thus, the MFCQ is verified, implying Abadie's CQ. 
At last, assume that (iii) is verified. Similarly to the case (ii) we can show that the direction $\boldsymbol{d}=$ $\left(\alpha \boldsymbol{x}, \mathbf{0}^{m}, \mathbf{0}^{r}, \beta[\boldsymbol{u}(\boldsymbol{x})-\boldsymbol{u}]\right)$ satisfies the requirements of MFCQ for some $\alpha>0, \beta \geq 0$, where $\boldsymbol{u}(\boldsymbol{x})$ is the equilibrium displacement, corresponding to $\boldsymbol{x}$.

Naturally, all three assumptions of Proposition 4.9 are violated by Example 4.8.

It is interesting to note that topology optimization problems for trusses without unilateral constraints are always qualified in the sense of Mangasarian-Fromowitz; it is probably even more interesting to see that the violation of MFCQ may happen even for "nice" feasible points that verify a strict complementarity assumption for MPEC problems (like the point considered in Example 4.8).

\subsection{Optimality conditions for $(\mathcal{W})$}

Motivated by the description of the feasible sets of the approximating problems $\left(\mathcal{W}^{\varepsilon}\right), \varepsilon>0$, in terms of differentiable inequalities which lead to at least Fritz-John necessary optimality conditions (see Example 4.8 and Proposition 4.9), we may use the same description with $\varepsilon=0$ in order to develop non-smooth necessary optimality conditions for $(\mathcal{W})$. The biggest difficulty we encounter is the loss of continuity (not to mention differentiability) of the complementary energy function $\mathcal{E}$. Indeed, if we look at the constraint involving $\mathcal{E}$ :

$$
\mathcal{E}(\boldsymbol{x}, \boldsymbol{s}, \boldsymbol{\lambda})+\Pi(\boldsymbol{x}, \boldsymbol{u})-\boldsymbol{f}^{t} \boldsymbol{u} \leq 0,
$$

we note that the function on the left-hand side of the inequality is neither Lipschitz continuous nor convex, and therefore the classic subdifferentials of such functions are not defined. On the other hand, we may use the structure of this function: it is continuously differentiable everywhere except when $\boldsymbol{x} \in \partial \mathbb{R}_{+}^{m}$, and it is a sum of convex and Lipschitz continuous functions. Therefore, the notion of limiting subdifferential $\partial_{a}$ is well defined for such functions (see [Mor76]). In particular, it holds that

$$
\partial_{a}\left[\mathcal{E}(\boldsymbol{x}, \boldsymbol{s}, \boldsymbol{\lambda})+\Pi(\boldsymbol{x}, \boldsymbol{u})-\boldsymbol{f}^{t} \boldsymbol{u}\right]=\partial_{a} \mathcal{E}(\boldsymbol{x}, \boldsymbol{s}, \boldsymbol{\lambda})+\nabla\left[\Pi(\boldsymbol{x}, \boldsymbol{u})-\boldsymbol{f}^{t} \boldsymbol{u}\right] .
$$

As a result, we obtain the following non-smooth Fritz-John type optimality conditions.

Proposition 4.10. Let $(\boldsymbol{x}, \boldsymbol{s}, \boldsymbol{\lambda}, \boldsymbol{u})$ be a point of local minimum for $(\mathcal{W})$. To simplify notation we write all inequality and equality constraints of $(\mathcal{W})$, except the relaxed equilibrium, constraint in the form:

$$
\begin{aligned}
& \mathrm{A}_{i}\left(\boldsymbol{x}^{t}, \boldsymbol{s}^{t}, \boldsymbol{\lambda}^{t}, \boldsymbol{u}^{t}\right)^{t} \leq \boldsymbol{b}_{i}, \\
& \mathrm{~A}_{e}\left(\boldsymbol{x}^{t}, \boldsymbol{s}^{t}, \boldsymbol{\lambda}^{t}, \boldsymbol{u}^{t}\right)^{t}=\boldsymbol{b}_{e},
\end{aligned}
$$

where $\mathrm{A}_{i} \in \mathbb{R}^{N_{i} \times(m+m+r+n)}, \mathrm{A}_{e} \in \mathbb{R}^{N_{e} \times(m+m+r+n)}, \boldsymbol{b}_{i} \in \mathbb{R}^{N_{i}}$, and $\boldsymbol{b}_{e} \in \mathbb{R}^{N_{e}}$ are matrices and vectors of appropriate sizes. Then, the non-smooth Fritz-John optimality conditions hold at $(\boldsymbol{x}, \boldsymbol{s}, \boldsymbol{\lambda}, \boldsymbol{u})$, that is, there are vectors $\boldsymbol{\mu}_{i} \in \mathbb{R}_{+}^{N_{i}}, \boldsymbol{\mu}_{e} \in \mathbb{R}^{N_{e}}$, and numbers $\mu_{0}, \mu \in \mathbb{R}_{+}$not all equal to zero such that:

$$
\begin{aligned}
\mathbf{0}^{m+m+r+n} & \left.\in \mu_{0} \nabla w(\boldsymbol{x})+\mathrm{A}_{i}^{t} \boldsymbol{\mu}_{i}+\mathrm{A}_{e}^{t} \boldsymbol{\mu}_{e}+\mu\left[\partial_{a} \mathcal{E}(\boldsymbol{x}, \boldsymbol{s}, \boldsymbol{\lambda})+\nabla\left(\Pi(\boldsymbol{x}, \boldsymbol{u})-\boldsymbol{f}^{t} \boldsymbol{u}\right)\right)\right], \quad \text { and } \\
0 & =\boldsymbol{\mu}_{i}^{t}\left[\mathrm{~A}_{i}\left(\boldsymbol{x}^{t}, \boldsymbol{s}^{t}, \boldsymbol{\lambda}^{t}, \boldsymbol{u}^{t}\right)^{t}-\boldsymbol{b}_{i}\right] .
\end{aligned}
$$

In general, we cannot expect the KKT conditions to be satisfied at every point of local minimum, because the problem $(\mathcal{W})$ is usually much less regular than its approximation $\left(\mathcal{W}^{\varepsilon}\right), \varepsilon>0$, and even the latter problem may violate the standard nonlinear programming constraint qualifications (see Example 4.8). In fact, in Problem 2, Subsection 5.2, we obtained a locally optimal solution that satisfies the system (7) only with $\mu_{0}=0$. It is sad to note that this example does not contain any contact conditions, and the optimal solution we obtained is non-singular (in particular, no bars were removed), yet it is only a FJ point in our formulation. On the positive side, at least if unilateral condition are absent, the conditions (7) imply the fulfillment of the KKT conditions for a related optimization problem that has a clear engineering interpretation. Namely, the stationary point obtained is a KKT point for a "semi-fixed topology" optimization problem, in which the given subset of the bars is removed from the ground structure; formally, the following result holds.

Proposition 4.11. Assume that the unilateral constraints are absent and that the point $(\hat{\boldsymbol{x}}, \hat{\boldsymbol{s}}, \hat{\boldsymbol{u}}) \in \mathbb{R}_{+}^{m} \times$ $\mathbb{R}^{m} \times \mathbb{R}^{n}$ satisfies the FJ optimality conditions (7). Let $\hat{\mathcal{I}}=\left\{i=1, \ldots, m \mid \hat{x}_{i}=0\right\}$. Then, the point 
$(\hat{\boldsymbol{x}}, \hat{\boldsymbol{s}}, \hat{\boldsymbol{u}})$ is a KKT-point for the following problem:

$$
(\hat{\mathcal{W}})\left\{\begin{array}{l}
\min _{(\boldsymbol{x}, \boldsymbol{s}, \boldsymbol{u})} w(\boldsymbol{x}) \\
\text { s.t. } \mathrm{B}^{t} \boldsymbol{s}=\boldsymbol{f}, \\
\\
\mathcal{E}(\boldsymbol{x}, \boldsymbol{s}, \boldsymbol{\lambda})+\Pi(\boldsymbol{x}, \boldsymbol{u})-\boldsymbol{f}^{t} \boldsymbol{u}=0, \\
\quad \underline{\sigma}_{i} x_{i} \leq s_{i} \leq \bar{\sigma}_{i} x_{i}, \quad i \in\{1, \ldots, m\} \backslash \hat{\mathcal{I}}, \\
x_{i}=s_{i}=0, \quad i \in \hat{\mathcal{I}} .
\end{array}\right.
$$

Proof. Clearly the point $(\hat{\boldsymbol{x}}, \hat{\boldsymbol{s}}, \hat{\boldsymbol{u}})$ is feasible in the problem $(\hat{\mathcal{W}})$. Furthermore, it is easy to check that the feasible set of the problem $(\hat{\mathcal{W}})$ verifies a Mangasarian-Fromowitz type constraint qualification at $(\hat{\boldsymbol{x}}, \hat{\boldsymbol{s}}, \hat{\boldsymbol{u}})$ [one can, for example, take the direction $\boldsymbol{d}=(\hat{\boldsymbol{x}}, \mathbf{0},-\boldsymbol{u}) \in \mathbb{R}_{+}^{m} \times \mathbb{R}^{m} \times \mathbb{R}^{n}$ to verify that], and thus the FJ conditions $(7)$ [that can be viewed as FJ conditions for $(\hat{\mathcal{W}})$ ] also imply the KKT conditions.

There are of course other approaches to optimality conditions for MPEC. For example, [OKZ98, Theorem 7.2] establishes non-smooth KKT-type conditions for a problem rather similar to $(\mathcal{W})$. However, the strong regularity condition on the lower-level problem assumed in [OKZ98, Theorem 7.2] is violated by our problem, because the displacements $\boldsymbol{u}$ are in general not uniquely determined for designs $\boldsymbol{x} \in \partial \mathbb{R}_{+}^{m}$.

\subsection{Global convergence}

Convergence of globally optimal solutions to relaxed weight minimization problems with stress constraints $\left(\mathcal{W}^{\varepsilon}\right)$ towards globally optimal solutions to the limiting problem $(\mathcal{W})$ as $\varepsilon \downarrow 0$ follows easily, given the results of the previous subsections.

Proposition 4.12. Consider a positive sequence $\left\{\varepsilon_{k}\right\}$ converging to zero. Let $\left\{\left(\boldsymbol{x}_{\varepsilon_{k}}, \boldsymbol{s}_{\varepsilon_{k}}, \boldsymbol{\lambda}_{\varepsilon_{k}}, \boldsymbol{u}_{\varepsilon_{k}}\right)\right\}$ be a corresponding sequence of globally optimal solutions to $\left\{\left(\mathcal{W}^{\varepsilon_{k}}\right)\right\}$. Then, an arbitrary limit point of this sequence is a globally optimal solution to the limiting problem $(\mathcal{W})$.

Proof. That globally optimal solutions to the sequence of problems $\left\{\left(\mathcal{W}^{\varepsilon_{k}}\right)\right\}$ exist follows by the coercivity of the objective w.r.t. the design variables, Proposition 4.7, and Weierstrass' Theorem).

Without any loss of generality, assume that $\lim _{k \rightarrow+\infty}\left(\boldsymbol{x}_{\varepsilon_{k}}, \boldsymbol{s}_{\varepsilon_{k}}, \boldsymbol{\lambda}_{\varepsilon_{k}}, \boldsymbol{u}_{\varepsilon_{k}}\right)=(\widetilde{\boldsymbol{x}}, \widetilde{\boldsymbol{s}}, \widetilde{\boldsymbol{\lambda}}, \widetilde{\boldsymbol{u}})$. Then, owing to Proposition 4.5 , the point $(\widetilde{\boldsymbol{x}}, \widetilde{\boldsymbol{s}}, \widetilde{\boldsymbol{\lambda}}, \widetilde{\boldsymbol{u}})$ is feasible in $(\mathcal{W})$. Together with Remark 4.4 and the continuity of the objective functional this proves the claim.

In general, the displacement component $\left\{\boldsymbol{u}_{k}\right\}$ of the sequence of global optimal solutions we study in Proposition 4.12 need not to have any limit points. However, we may use the fact that our objective function is independent of the displacements and utilize Proposition 4.6 to establish the following result.

Proposition 4.13. Consider a positive sequence $\left\{\varepsilon_{k}\right\}$ converging to zero. Let $\left\{\left(\boldsymbol{x}_{\varepsilon_{k}}, \boldsymbol{s}_{\varepsilon_{k}}, \boldsymbol{\lambda}_{\varepsilon_{k}}, \boldsymbol{u}_{\varepsilon_{k}}\right)\right\}$ be a corresponding sequence of globally optimal solutions to $\left\{\left(\mathcal{W}^{\varepsilon_{k}}\right)\right\}$. Then, an arbitrary limit point $\left(\boldsymbol{x}_{0}, \boldsymbol{s}_{0}, \boldsymbol{\lambda}_{0}\right)$ of the sequence $\left\{\left(\boldsymbol{x}_{\varepsilon_{k}}, \boldsymbol{s}_{\varepsilon_{k}}, \boldsymbol{\lambda}_{\varepsilon_{k}}\right)\right\}$ (and there is at least one) corresponds to some globally optimal solution $\left(\boldsymbol{x}_{0}, \boldsymbol{s}_{0}, \boldsymbol{\lambda}_{0}, \boldsymbol{u}_{0}\right)$ to the limiting problem $(\mathcal{W})$.

Proof. Similar to the proof of Proposition 4.12, but uses Proposition 4.6 instead of Proposition 4.5.

\subsection{Convergence of stationary points}

The main result of this paper, Theorem 4.14, uses the fact that stress constraints are imposed. Furthermore, we need to make an assumption that the sequence of displacements $\left\{\boldsymbol{u}^{\varepsilon}\right\}$ produced by the smoothing procedure is bounded as $\varepsilon \downarrow 0$. We cannot guarantee the latter property without imposing explicit bounds on the displacements; however, our computational experience with the smoothing approach we introduce in this paper confirms that convergence of displacements takes place in practice. In any case, Proposition 4.3 asserts that it is at least possible to approximate every equilibrium state using the relaxation approach we propose; this is contrary to traditional $\varepsilon$-relaxation, where some equilibrium displacements cannot be approximated.

Theorem 4.14. Consider a positive sequence $\left\{\varepsilon_{k}\right\}$ converging to zero. Let $\left\{\left(\boldsymbol{x}_{\varepsilon_{k}}, \boldsymbol{s}_{\varepsilon_{k}}, \boldsymbol{\lambda}_{\varepsilon_{k}}, \boldsymbol{u}_{\varepsilon_{k}}\right)\right\}$ be a sequence of KKT-points to $\left\{\left(\mathcal{W}^{\varepsilon_{k}}\right)\right\}$. Then, every limit point of this sequence is feasible in the limiting problem $(\mathcal{W})$, and in addition it verifies the non-smooth FJ stationarity conditions (7). 
Proof. Without loss of generality we assume that $\left\{\left(\boldsymbol{x}_{\varepsilon_{k}}, \boldsymbol{s}_{\varepsilon_{k}}, \boldsymbol{\lambda}_{\varepsilon_{k}}, \boldsymbol{u}_{\varepsilon_{k}}\right)\right\} \rightarrow(\boldsymbol{x}, \boldsymbol{s}, \boldsymbol{\lambda}, \boldsymbol{u})$ as $k$ converges to infinity. Owing to Proposition 4.5, the point $(\boldsymbol{x}, \boldsymbol{s}, \boldsymbol{\lambda}, \boldsymbol{u})$ is feasible in $(\mathcal{W})$.

The stress constraints imply that the gradients $\nabla \mathcal{E}\left(\boldsymbol{x}_{\varepsilon_{k}}, \boldsymbol{s}_{\varepsilon_{k}}, \boldsymbol{\lambda}_{\varepsilon_{k}}\right)$ are uniformly bounded for $k=1,2, \ldots$ Therefore, the sequence $\left\{\nabla \mathcal{E}\left(\boldsymbol{x}_{\varepsilon_{k}}, \boldsymbol{s}_{\varepsilon_{k}}, \boldsymbol{\lambda}_{\varepsilon_{k}}\right)\right\}$ has at least one limit point that by definition is a member of $\partial_{a} \mathcal{E}(\boldsymbol{x}, \boldsymbol{s}, \boldsymbol{\lambda})$. It is now an easy exercise to verify that the point $(\boldsymbol{x}, \boldsymbol{s}, \boldsymbol{\lambda}, \boldsymbol{u})$ satisfies the system $(7)$.

Again, the optimality conditions we obtain in Theorem 4.14 are rather weak, but we cannot expect more from points of local minima for $(\mathcal{W})$ in general; see the discussion in Section 4.5.

\section{$5 \quad$ Numerical experiments}

While a substantial amount of theoretical studies of topology optimization problems for trusses including unilateral frictionless contact has been carried out (see, e.g., [BTKNZ99, PaP02, EPP03]), surprisingly little numerical experience has been reported. Therefore we use a comprehensive numerical study of Stolpe [Sto04] (who was interested in finding globally optimal solutions using a branch-and-cut algorithm) as a rich and authoritative source of benchmark problems, unfortunately however for trusses without contact. We also compare our algorithm against a few tests of "classic" MPEC algorithms (implicit programming-based algorithm, IMPA, [LPR96, Section 6.3], and penalty interior point algorithm, PIPA, [LPR96, Section 6.1]), MMA [Sva87] (see also [Sva02]), as well as the smoothing algorithm [FJQ99], made by Hilding [HKP99, Hil00]. Unfortunately, the latter studies are not concerned with topology optimization (i.e., a strictly positive bound on the bar volumes is imposed) and stress constraints are not included.

Below we present some preliminary numerical experience with an academic implementation of our approximation method.

\subsection{Implementation issues}

A sequence of smooth optimization subproblems $\left\{\left(\mathcal{W}^{\varepsilon_{k}}\right)\right\}$ has been solved using the SQP-solver SNOPT [GMS02]. The optimal solution obtained at step $k$ was used as a starting point for the step $k+1$. We used a simple update rule for $\varepsilon: \varepsilon_{k+1}=\gamma \varepsilon_{k}$, where $\gamma \in[0.25,0.75]$.

The biggest computational difficulty we have noted is that the projected gradient of the potential energy with respect to displacements is close to zero for all points feasible in $\left(\mathcal{W}^{\varepsilon}\right)$ when $\varepsilon$ is small, resulting in rather slow progress of the optimization procedure based on the first order information only. The use of second order information in this case seems essential for improving the performance.

Another problem is that the complementary energy has a rather unusual scaling when the design variables $\boldsymbol{x}$ are close to the boundary $\partial \mathbb{R}_{+}^{m}$. While we obtained satisfactory results with automatic scaling in SNOPT, a specific scaling of the relaxed equilibrium constraint may be necessary for more robust convergence of the algorithm.

\subsection{Numerical results: topology optimization, contact-less case}

A number of "classic" weight minimization problems for trusses without frictionless contact but including stress, and possibly local buckling constraints and bounds on displacements have been solved to global optimality by Stolpe [Sto04]. We benchmark our relaxation algorithm against the results reported in the cited paper and find that in many cases our local algorithm is capable of finding globally optimal solutions. We keep the problem numbers assigned by Stolpe [Sto04] and report the results we obtained on a subset of these problems in Tables 1 (only stress and/or displacement constraints) and 2 (stress and local buckling constraints).

Since we use a local algorithm to solve non-convex optimization problems, starting the optimization procedure from different starting points may result in obtaining different optimal solutions. We started the algorithm from the design obtained by uniformly distributing structural material among bars, and calculating the corresponding equilibrium forces/displacements.

Some comments are in order. In problems $24-26$ the number of bars in the structure is $m=10$, but the volumes of 4 of them are fixed, which leaves us only 6 design variables. In addition, these are the only problems with displacement constraints, and the optimal weight we report differs from the known globally optimal solution despite the small value of the relaxation parameter $\varepsilon$ we used. The reason for such a behaviour is that the potential energy $\Pi(\boldsymbol{x}, \boldsymbol{u})-\boldsymbol{f}^{t} \boldsymbol{u}$ becomes rather insensitive to some components of the displacements for designs $\boldsymbol{x}$ that are close to the boundary $\partial \mathbb{R}_{+}^{m}$. In problems 24-26 this allows the optimization procedure to choose displacements that are reasonably far from the equilibrium displacements (compared to the size of the relaxation parameter $\varepsilon$ ) but are feasible with respect to the imposed bounds on the displacements. (Recall that Proposition 4.6 does not guarantee the convergence of the displacements 


\begin{tabular}{rrrrrrl} 
Problem & $m$ & $n$ & $k$ & $w_{\text {our }}$ & $w_{[\text {Sto04] }}$ source \\
\hline 2 & 5 & 4 & 2 & 39.9856 & 33.5000 & {$[$ ChG97] } \\
5 & 4 & 2 & 2 & 185.597 & 185.667 & {$[$ Hob96] } \\
9 & 10 & 8 & 1 & 4896.95 & 4898.31 & {$[$ ScF74] } \\
11 & 10 & 8 & 1 & 1583.99 & 1584.00 & {$[$ ScF74] } \\
13 & 10 & 8 & 1 & 4425.16 & 4426.52 & {$[$ ScF74] } \\
15 & 10 & 8 & 1 & 1655.99 & 1656.00 & {$[$ ScF74] } \\
${ }^{*} 17$ & 25 & 18 & 5 & 510.157 & 545.264 & {$[$ ScF74] } \\
18 & 10 & 8 & 1 & 1583.99 & 1584.00 & {$[$ ChG97] } \\
23 & 5 & 4 & 1 & 24.0000 & 24.0000 & {$[$ ChJ92] } \\
24 & $10(6)$ & 8 & 1 & 18199.4 & 18211.8 & {$[$ Kir90] } \\
25 & $10(6)$ & 8 & 1 & 20021.8 & 20035.3 & {$[$ Kir90] } \\
26 & $10(6)$ & 8 & 1 & 22799.7 & 22817.3 & {$[$ Kir90] } \\
27 & 10 & 8 & 1 & 1979.99 & 1980.00 & {$[$ GCY01] } \\
28 & 5 & 4 & 2 & 79.9713 & 79.9716 & {$[$ GCY01] } \\
\hline
\end{tabular}

Table 1: Results of numerical experiments: weight minimization under stress and/or displacement constraints

\begin{tabular}{rrrrrrl} 
Problem & $m$ & $n$ & $k$ & $w_{\text {our }}$ & $w_{[\text {Sto04] }}$ & source \\
\hline 5 & 4 & 2 & 2 & 408.312 & 408.628 & [Hob96] \\
27 & 10 & 8 & 1 & 8553.44 & 8553.44 & [GCY01] \\
28 & 5 & 4 & 2 & 105.831 & 105.831 & [GCY01] \\
\hline
\end{tabular}

Table 2: Results of numerical experiments: weight minimization under stress and local buckling constraints

as designs converge.) This may or may not be a problem in practice, depending on how stringent the displacement constraints are, if present. In particular, we guarantee the convergence of forces, and always keep the stress (and local buckling) constraints satisfied, which means that the structure will not suffer from destructive stresses. (Even though stress constraints are imposed not on the "equilibrium" stresses, stress bounds are usually chosen far from the point where plastic deformation occurs.) In any case, our algorithm successfully finds the optimal topology, which is of major importance in many applications.

In problem 17 our algorithm indeed finds a better solution to the classic 25-bar truss problem stated in [ScF74] than the one reported in [Sto04, ScF74]. The reason for this small victory of a local optimization algorithm over a global one is that the branch-and-cut method developed in [Sto04] may be applied only to problems with bounds imposed on all variables involved. In the original formulation of the problem 17 taken from [ScF74] there are no upper bounds on the volumes of the bars, and the optimal weight of the truss we obtained for the original formulation is 510.157. On the other hand, Stolpe [Sto04] imposes artificial bounds on the design variables for the branch-and-cut method to function, which leads to a globally optimal solution with the weight 545.264; in fact, the newly introduced bounds are inactive at the latter solution but owing to the non-convexity of the problem they cannot be safely removed without changing the optimal solution. The last comment about the problem 17 is that in the original formulation there are only 2 load scenarios and many linear constraints on the design variables related to the required symmetry of the truss. Instead, we consider all design variables to be independent and obtain a symmetric solution by introducing additional load cases.

\subsection{Numerical results: sizing optimization of trusses in contact}

Hilding et al. [HKP99] (see also Hilding [Hil00]) were interested in minimizing the maximal contact force, that is, to achieve as uniform contact pressures as possible. The formal problem statement can be written as follows:

$$
(\Lambda)\left\{\begin{array}{l}
\min _{\left(\boldsymbol{x}, \boldsymbol{s}, \boldsymbol{\lambda}, \boldsymbol{u}, \lambda_{\max }\right)} \lambda_{\max }, \\
\text { s.t. }(\boldsymbol{x}, \boldsymbol{s}, \boldsymbol{\lambda}, \boldsymbol{u}) \in \mathcal{F}(\boldsymbol{f}), \\
\quad \underline{x}_{i} \leq x_{i} \leq \bar{x}_{i}, \quad i=1, \ldots, m, \\
w(\boldsymbol{x}) \leq \bar{w}, \\
\lambda_{\ell} \leq \lambda_{\max }, \quad \ell=1, \ldots, r,
\end{array}\right.
$$

where $\underline{x}_{i}, \bar{x}_{i}, \bar{w}$ are given positive numbers, $i=1, \ldots, m$. In general, allowing lower bounds on the design variables to be zero results in an ill-posed optimization problem, unless bounds on the compliance of the 


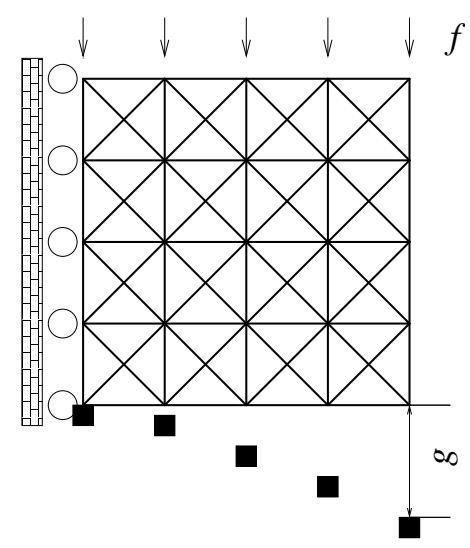

Figure 3: Test problem found in [Hil00]. $5 \times 5$ case is shown.

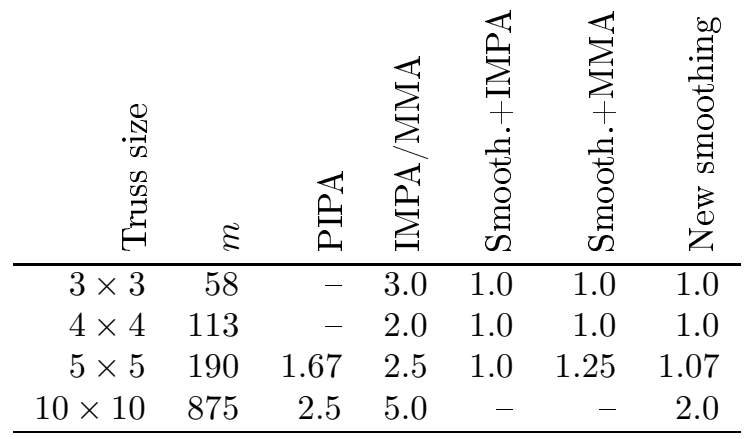

Table 3: Results of numerical experiments: contact force minimization.

structure or stress constraints are added (see [PaP02]).

The problem $(\Lambda)$ is thus not a topology optimization problem and does not suffer from the difficulties outlined in Section 4.1; in particular, the smoothing method of Facchinei [FJQ99] outlined in Section 3 is directly applicable to this problem (see [Hil00], where smoothing was used for "the heuristic avoiding of local minima") and we use it as one of the benchmarks for our new smoothing algorithm.

On some instances of the problem $(\Lambda)$ Hilding et al. [HKP99] also implemented and tested some classic MPEC algorithms (IMPA [LPR96, Section 6.3] and PIPA [LPR96, Section 6.1]) on the family of structures shown in Figure 3. Also, they tested on $(\Lambda)$ a very popular method in the structural optimization community: the method of moving asymptotes, MMA, [Sva87], even though it is not guaranteed to work on this problem.

We apply SNOPT to the following relaxation of the problem $(\Lambda)$ :

$$
\left(\Lambda^{\varepsilon}\right)\left\{\begin{array}{l}
\min _{\left(\boldsymbol{x}, \boldsymbol{s}, \boldsymbol{\lambda}, \boldsymbol{u}, \lambda_{\max }\right)} \lambda_{\max }, \\
\text { s.t. }(\boldsymbol{x}, \boldsymbol{s}, \boldsymbol{\lambda}, \boldsymbol{u}) \in \mathcal{F}^{\varepsilon}(\boldsymbol{f}), \\
\quad \underline{x}_{i} \leq x_{i} \leq \bar{x}_{i}, \quad i=1, \ldots, m, \\
w(\boldsymbol{x}) \leq \bar{w}, \\
\lambda_{\ell} \leq \lambda_{\max }, \quad \ell=1, \ldots, r,
\end{array}\right.
$$

where $\varepsilon>0$ is a relaxation parameter. We report the results we obtained for trusses of different sizes (see Figure 3 ) in Table 3 along with the results found in [HKP99, Hil00].

We report the size of the structure, the number of bars (design variables) and the optimal values obtained by PIPA and IMPA/MMA as reported in [HKP99] (the two latter algorithms are reported to produce the same optimal values); the optimal values produced by IMPA and MMA as applied to the smoothed MPEC using the methodology introduced in [FJQ99], as reported in [Hil00]; and the optimal values obtained using our new smoothing procedure. The "-" sign in the table columns means that the corresponding algorithm has not been applied to a given problem instance.

One can see that our algorithm favourably competes with classic MPEC algorithms on these tests. As 
we already mentioned, general MPEC algorithms cannot be applied to truss optimization problems if we remove strictly positive lower bounds on the design variables, i.e., consider topology optimization problems.

\section{Conclusions and further research}

In this paper we proposed a new algorithm for solving MPEC problems arising from the topology optimization of trusses with unilateral contact conditions. The algorithm is based on the approximation of topology optimization problems with sizing-type problems, where in addition we relax the equilibrium constraint. We studied the convergence of global optimal solutions and stationary points to approximating problems towards, respectively, globally optimal solutions and stationary points to the original, singular problem. We have also performed some numerical testing of the proposed method.

Many open problems remain. On the numerical side, we need a better implementation (probably utilizing second order information); also, a much more thorough numerical testing should be done, especially for trusses with unilateral contact. However, in our opinion, the most challenging task is to improve the optimality conditions we obtained in this paper. To do that, the comparative analysis of modern KKT-type optimality conditions for general MPEC problems (see, e.g., [FlK02a, FlK02b, FlK02c]) and the FJ-type optimality conditions we obtained needs to be performed. We hope to address these questions in our future research.

\section{References}

[Ach98] W. Achtziger. Multiple-load truss topology and sizing optimization: Some properties of minimax compliance. J. Optim. Theory Appl., 98(2):255-280, 1998.

[Ach03] W. Achtziger. On the optimality conditions and primal-dual methods for the detection of singular optima. In Carlo Cinquini, Marco Rovati, Paolo Venini, and Roberto Nascimbene, editors, Short Papers of The Fifth World Congress of Structural and Multidisciplinary Optimization, pages 1-2, 2003.

[AuF90] Jean-Pierre Aubin and Hélène Frankowska. Set-Valued Analysis. Birkhäuser Boston Inc., Boston, MA, 1990.

[BTKNZ99] A. Ben-Tal, M. Kočvara, A. Nemirovski, and J. Zowe. Free material design via semidefinite programming: the multiload case with contact conditions. SIAM J. Optim., 9(4):813-832, 1999.

[ChG97] G. Cheng and X. Guo. $\varepsilon$-relaxed approach in structural topology optimization. Struct. Optim., 13:258266, 1997.

[ChJ92] G. Cheng and Z. Jiang. Study on topology optimization with stress constraints. Eng. Opt., 20:129-148, 1992.

[DuB98] P. Duysinx and M. P. Bendsøe. Topology optimization with stress constraints. Int. J. Numer. Mech. Engrg., 43:1453-1478, 1998.

[DuS98] P. Duysinx and O. Sigmund. New developments in handling stress constraints in optimal material distribution. In Proc. 7th AIAA/USAF/NASA/ISSMO Symposium on Multidiciplinary Analysis and Optimization, pages 1501-1509, 1998.

[EPP03] Anton Evgrafov, Michael Patriksson, and Joakim Petersson. Stochastic structural topology optimization: Existence of solutions and sensitivity analyses. ZAMM Z. angew. Math. Mech., 83(7):479-492, 2003.

[Evg04] Anton Evgrafov. On globally stable singular topologies. Struct. Multidisc. Optim., 2004. Published online 14 October 2004, DOI 10.1007/s00158-004-0428-6.

[FaP03] Francisco Facchinei and Jong-Shi Pang. Finite-dimensional variational inequalities and complementarity problems. Vol. I. Springer Series in Operations Research. Springer-Verlag, New York, 2003.

[FJQ99] Francisco Facchinei, Houyuan Jiang, and Liqun Qi. A smoothing method for mathematical programs with equilibrium constraints. Math. Program., 85(1, Ser. A):107-134, 1999.

[FIK02a] Michael L. Flegel and Christian Kanzow. An Abadie-type constraint qualification for mathematical problems with equilibrium constraints. Preprint, University of Würzburg, Institute of Applied Mathematics and Statistics, November 2002.

[FlK02b] Michael L. Flegel and Christian Kanzow. On the Guignard constraint qualification for mathematical problems with equilibrium constraints. Preprint, University of Würzburg, Institute of Applied Mathematics and Statistics, October 2002.

[FIK02c] Michael L. Flegel and Christian Kanzow. Optimality conditions for mathematical programs with equilibrium constraints: Fritz John and Abadie-type approaches. Preprint, University of Würzburg, Institute of Applied Mathematics and Statistics, May 2002.

[GCY01] X. Guo, G. Cheng, and K. Yamazaki. A new approach for the solution of singular optima in truss topology optimization with stress and local buckling constraints. Struct. and Multidisc. Optim., 22:364$372,2001$. 
[GMS02] Philip E. Gill, Walter Murray, and Michael A. Saunders. SNOPT: an SQP algorithm for large-scale constrained optimization. SIAM J. Optim., 12(4):979-1006, 2002.

[Hil00] Daniel Hilding. A heuristic smoothing procedure for avoiding local optima in optimization of structures subject to unilateral constraints. Struct. Multidisc. Optim., 20(1):29-36, Aug 2000.

[HKP99] Daniel Hilding, Anders Klarbring, and Jong-Shi Pang. Minimization of maximum unilateral force. Comput. Methods Appl. Mech. Engrg., 177(3-4):215-234, 1999.

[Hob96] A. S. Hoback. Optimization of singular problems. Struct. Optim., 12:93-97, 1996.

[Kir90] U. Kirsch. On singular topologies in optimum structural design. Struct. Optim., 2(3):133-142, 1990.

[LiM97] M. B. Lignola and J. Morgan. Stability of regularized bilevel programming problems. J. Optim. Theory Appl., 93(3):575-596, 1997.

[LPR96] Zhi-Quan Luo, Jong-Shi Pang, and Daniel Ralph. Mathematical Programs with Equilibrium Constraints. Cambridge University Press, Cambridge, 1996.

[Mic04] A. G. M. Michell. The limits of economy of material in frame structures. Phil. Mag., 8, 1904.

[Mor76] B. Sh. Mordukhovich. Maximum principle in the problem of time optimal response with nonsmooth constraints. Prikl. Mat. Meh., 40(6):1014-1023, 1976.

[OKZ98] Jiři Outrata, Michal Kočvara, and Jochem Zowe. Nonsmooth Approach to Optimization Problems with Equilibrium Constraints. Kluwer Academic Publishers, Dordrecht, 1998.

[Out94] J. V. Outrata. On optimization problems with variational inequality constraints. SIAM Journal on Optimization, 4:340-357, 1994.

[OuZ95] J. Outrata and J. Zowe. A numerical approach to optimization problems with variational inequality constraints. Mathematical Programming, 68:105-130, 1995.

[PaP02] Michael Patriksson and Joakim Petersson. Existence and continuity of optimal solutions to some structural topology optimization problems including unilateral constraints and stochastic loads. ZAMM Z. Angew. Math. Mech., 82(7):435-459, 2002.

[Pet01] Joakim Petersson. On continuity of the design-to-state mappings for trusses with variable topology. Internat. J. Engrg. Sci., 39(10):1119-1141, 2001.

[RoB94] G. I. N. Rozvany and T. Birker. On singular topologies in exact layout optimization. Struct. Optim., 8:228-235, 1994.

[ScF74] L. A. Schmit and B. Farshi. Some approximation consepts for structural synthesis. AIAA Journal, 12(5):692-699, 1974.

[Sch01] Stefan Scholtes. Convergence properties of a regularization scheme for mathematical programs with complementarity constraints. SIAM J. Optim., 11(4):918-936 (electronic), 2001.

[Sto04] M. Stolpe. Global optimization of minimum weight truss topology problems with stress, displacement, and local buckling constraints using branch-and-bound. Internat. J. Numer. Methods Engrg., 61(8):1270-1309, 2004.

[StS01] M. Stolpe and K. Svanberg. On trajectories of the epsilon-relaxation approach for stress constrained truss topology optimization. Struct. and Multidisc. Optim., 21(2):140-151, 2001.

[StS03] M. Stolpe and K. Svanberg. A note on stress-constrained truss topology optimization. Struct. and Multidisc. Optim., 25(1):62-64, 2003.

[StS04] M. Stolpe and K. Svanberg. A stress-constrained truss-topology and material-selection problem that can be solved by linear programming. Struct. Multidiscip. Optim., 27(1-2):126-129, 2004.

[Sva87] Krister Svanberg. The method of moving asymptotes - a new method for structural optimization. Internat. J. Numer. Methods Engrg., 24(2):359-373, 1987.

[Sva02] Krister Svanberg. A class of globally convergent optimization methods based on conservative convex separable approximations. SIAM J. Optim., 12(2):555-573, 2002.

[SvG68] G. Sved and Z. Ginos. Structural optimization under multiple loading. Int. J. Mech. Sci., 10:803-805, 1968. 